\title{
Three Efficient Numerical Models to Analyse the Step Problem in Shallow Water
}

\author{
E. G. A. Costa $^{1 *}$, J. A. F. Santiago ${ }^{1}$, L. M. C. Godinho ${ }^{2}$, L. C. Wrobel ${ }^{3}$, W. J. Mansur ${ }^{1}$ \\ ${ }^{1}$ COPPE/UFRJ - Program of Civil Engineering \\ Federal University of Rio de Janeiro, CP 68506, CEP 21945-970, Rio de Janeiro, RJ, Brazil \\ ${ }^{2}$ CICC - Department of Civil Engineering \\ University of Coimbra, 3030-788, Coimbra, Portugal \\ ${ }^{3}$ Brunel University London, Institute of Materials and Manufacturing \\ Uxbridge, UB8 3PH, England \\ *contact author: Edmundo Costa (edmundo_costa@coc.ufrj.br)
}

\begin{abstract}
In this paper, the problem of acoustic wave propagation in a waveguide of infinite extent is modelled, taking into account constant depth in each section of the sea. Efficient numerical strategies in the frequency domain are addressed to investigate two-dimensional acoustic wave propagation in a shallow water configuration, considering a step in the rigid bottom and a flat free surface. The time domain responses are obtained by means of an inverse Fast Fourier Transform (FFT) of results computed in the frequency domain. The numerical approaches used here are based on the Boundary Element Method (BEM) and the Method of Fundamental Solutions (MFS). In the numerical models only the inclined or vertical interface between the sub-regions of different depth are discretized, as Green's functions that take into account the presence of free and rigid surfaces are used. These Green's functions are obtained either by eigenfunction expansion or by Ewald's method. A detailed discussion on the performance of these formulations is carried out, with the aim of finding an efficient numerical formulation to solve the step problem in shallow water.
\end{abstract}

Keywords: Boundary Element Method, Method of Fundamental Solutions, Green's functions, Ewald's method, Shallow water 


\section{INTRODUCTION}

Many analytical and numerical methods have been developed to simulate and analyse underwater acoustic wave propagation. The book by Jensen et al. [1] discusses in detail the different methodologies applied to solve the problem of wave propagation in acoustic environments that have interested many researchers over past decades. Some of the wellknown methods are based on the acoustic ray theory [2], the normal modes method [3] and the parabolic equation [4].

A variety of numerical models has also been developed based on well-established approaches such as the finite difference, finite element and boundary element methods. Of these, the Boundary Element Method (BEM) permits an efficient analysis of underwater acoustic problems with complex shapes and complicated boundary conditions. The BEM has a number of advantages over other numerical methods [5], such as: it is very well suited for modeling homogeneous unbounded domains since it automatically satisfies the Sommerfeld radiation condition and thus involves a more compact description of the acoustic medium, requiring only the discretization of the problem boundaries, which considerably reduces the size of the final linear system of equations. However, the application of the boundary integral equation is often limited by the requirement of prior knowledge of the fundamental solutions and the appearance of singular or hyper-singular integrals in its formulation.

Another difficulty of the BEM in the analysis of acoustic wave propagation in shallow water occurs when more complex geometries are considered, requiring large discretization schemes. One way of avoiding this large discretization is by using Green's functions which directly satisfy the boundary conditions on the flat free surface and the rigid bottom of the ocean. Such Green's functions can be constructed using the image-source technique, but this leads to very slowly convergent series [6, 7]. An alternative to improve the convergence of the series is to build a Green's function in the form of eigenfunction expansions, the so-called normal mode solution. This function is also an infinite series but if only the evanescent modes are considered and there are no propagating modes, the series becomes rapidly convergent owing to the exponentially decaying terms for the evanescent modes. In spite of that, the convergence problems of this series still remain when the source and the field points are positioned along the same vertical alignment $[8,9]$.

Linton [10, 11] and Papanicolaou [12] discuss mathematical techniques for accelerating slowly convergent series and show that the Ewald's method is able to provide dramatic 
improvements in the speed of convergence, particularly when the source and field points are located along the same vertical line. This method has been successfully implemented in the boundary integral equation formulation by Venakides et al. [13], for the calculation of electromagnetic scattering of photonic crystals.

Santiago and Wrobel $[8,9]$ discussed the implementation of Ewald's method in a BEM model for underwater acoustics. They compared the convergence of Ewald's method with that of eigenfunction expansions, showing a substantial reduction in the number of terms necessary for convergence of the series, particularly when the source and field points are positioned along the same vertical line. In the present paper, for the first time, Ewald's method is fully implemented in a BEM underwater acoustics model in which a vertical interface is discretized, significantly improving the performance of the method. The integration of the resulting singular integrals is also discussed in the paper.

In recent years, meshless methods have attracted great interest of scientists and researchers. The Method of Fundamental Solution (MFS) is one of these methods and it has been applied with success for scattering or radiation problems. Mathematically, the MFS is a very simple technique and it is also based on the prior knowledge of fundamental solutions, but not requiring the numerical and analytical integrations that need to be performed in the BEM. One disadvantage of the MFS is the determination of the position of the pseudoboundary on which the singularities are placed. Karageorghis [14] has proposed a simple algorithm for estimating an optimal pseudo-boundary for certain boundary value problems. Costa et al. $[15,16]$ have shown that, despite its simplicity, the MFS is a very interesting tool to efficiently predict wave acoustic propagation in shallow water.

In this paper, the Boundary Element Method and the Method of Fundamental Solutions are used to analyse, in the frequency domain, the two-dimensional acoustic wave propagation in a shallow water configuration, considering a step up on the bottom of the sea. Time domain signals are computed by means of an inverse fast Fourier transform of the numerical results in the frequency domain. Appropriate Green's functions are used limiting the number of discretized surfaces and consequently reducing the computational cost of the proposed models. These models are developed by using a sub-region technique, where only the inclined or vertical interface between the sub-regions of different depth has to be discretized. These Green's functions are obtained either by eigenfunction expansion or by Ewald's method. A set of numerical examples is performed in order to demonstrate the efficiency of the proposed models in the analysis of acoustic wave propagation in shallow water problems containing a 
step on the seabed. In addition, a detailed discussion on the performance of these formulations is carried out, with the aim of finding an efficient formulation to solve the acoustic step problem in shallow water in the frequency domain.

\section{GOVERNING EQUATION OF THE PROBLEM}

The problem of two-dimensional acoustic wave propagation in a region $\Omega$ of infinite extent in the longitudinal z-direction is analysed, taking into account the presence of a step up on the bottom of the sea, as shown in Fig. 1. If the velocity of sound is constant, the source of acoustic disturbance is time-harmonic and the medium in the absence of perturbations is quiescent, the problem is governed by the Helmholtz equation which can be written as:

$\nabla^{2} p(\mathbf{x})+k^{2} p(\mathbf{x})=-Q \delta\left(\mathbf{x}-\xi^{s}\right)$ in $\Omega$,

where $\nabla^{2}=\frac{\partial}{\partial x^{2}}+\frac{\partial}{\partial y^{2}} ; p(\mathbf{x})$ is the acoustic pressure; $Q$ is the magnitude of the acoustic, sound-emitting source $\xi^{s}$ located at $\left(x_{\xi^{s}}, y_{\xi^{s}}\right) ; \mathbf{x}$ is the observation point located at $(x, y)$, $\delta\left(\mathbf{x}-\xi^{s}\right)$ is the Dirac delta function, and $k=2 \pi f / c$ is the wave number, with $f$ being the excitation frequency and $c$ the sound propagation velocity.

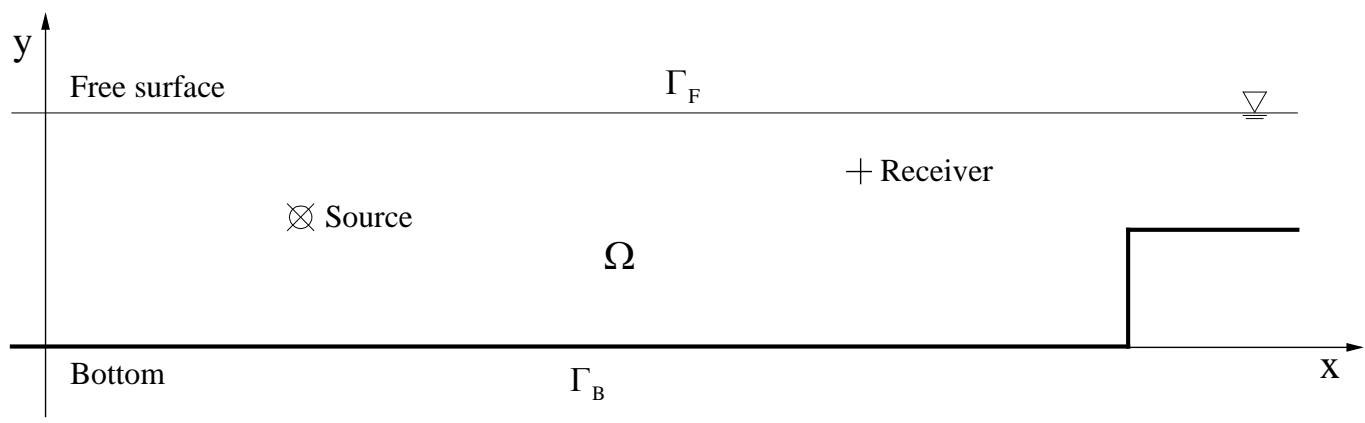

Figure 1: Geometry of the problem.

The boundary conditions for the above described problem are given by:

- Dirichlet condition

$$
p(\mathbf{x})=0 \quad \text { in } \Gamma_{\mathrm{F}}
$$


- Neumann condition

$$
\nu_{n}(\mathbf{x})=\frac{\mathrm{i}}{\omega \rho} \frac{\partial p(\mathbf{x})}{\partial \mathbf{n}}=0 \quad \text { in } \Gamma_{\mathrm{B}}
$$

where $\omega=2 \pi f$ is the angular frequency, $\rho$ is the density, $\mathbf{n}$ is the unit outward normal vector, $\mathrm{i}=\sqrt{-1}$ and $\Gamma_{\mathrm{F}}$ and $\Gamma_{\mathrm{B}}$ are the free and bottom surfaces, respectively.

\section{GREEN'S FUNCTIONS}

The Green's functions for the BEM and MFS models are developed using two numerical approaches. The first makes use of the eigenfunction expansion based on a combination of normal modes; and the second uses an alternative form based on the Ewald's method to improve the convergence of the series, particularly when the source and the receiver are positioned in the same vertical alignment. Both Green's functions exactly satisfy the boundary conditions on the free surface and the flat rigid bottom boundaries of the waveguide.

\subsection{Eigenfunction expansion}

The Green's function designated as $G_{\mathrm{M}}(\boldsymbol{\xi}, \mathbf{x})$ directly satisfies the boundary conditions on the flat rigid bottom and the free surface. In terms of normal modes [17], the Green's function can be written as:

$$
G_{\mathrm{M}}(\boldsymbol{\xi}, \mathbf{x})=\frac{\mathrm{i}}{H} \sum_{m=1}^{\infty} \sin \left[k_{y m}\left(Y_{\mathrm{F}}-y_{\xi}\right)\right] \sin \left[k_{y m}\left(Y_{\mathrm{F}}-y\right)\right] \frac{e^{\mathrm{i} k_{x m}\left|x-x_{\xi}\right|}}{k_{x m}}
$$

The derivatives with respect to $x$ and $y$ are:

$$
\begin{aligned}
& \frac{\partial G_{\mathrm{M}}(\boldsymbol{\xi}, \mathbf{x})}{\partial x}=-\frac{1}{H} \operatorname{sgn}\left(x-x_{\xi}\right) \sum_{m=1}^{\infty} \sin \left[k_{y m}\left(Y_{\mathrm{F}}-y_{\xi}\right)\right] \sin \left[k_{y m}\left(Y_{\mathrm{F}}-y\right)\right] e^{\mathrm{i} k_{x m}\left|x-x_{\xi}\right|}, \\
& \frac{\partial G_{\mathrm{M}}(\xi, \mathbf{x})}{\partial y}=-\frac{\mathrm{i}}{H} \sum_{m=1}^{\infty} k_{y m} \sin \left[k_{y m}\left(Y_{\mathrm{F}}-y_{\xi}\right)\right] \cos \left[k_{y m}\left(Y_{\mathrm{F}}-y\right)\right] \frac{e^{\mathrm{i} k_{x m}\left|x-x_{\xi}\right|}}{k_{x m}},
\end{aligned}
$$


where the source point $\xi$ is located at $\left(x_{\xi}, y_{\xi}\right)$, and $H$ is the depth of the waveguide $\left(Y_{\mathrm{F}}-Y_{\mathrm{B}}\right)$, as defined in Fig. 2. The parameters $k_{x m}=\sqrt{k^{2}-k_{y m}^{2}}$ and $k_{y m}=\left(m-\frac{1}{2}\right) \frac{\pi}{H}$ are horizontal and vertical wavenumbers, respectively.

It is important to note that the exponential term in Eqs. (4), (5) and (6) makes the convergence of the Green's function decrease rapidly when $k_{x m}$ becomes an imaginary number (evanescent modes), but when the exponential term is equal to 1.00 , the convergence of the series is much slower.

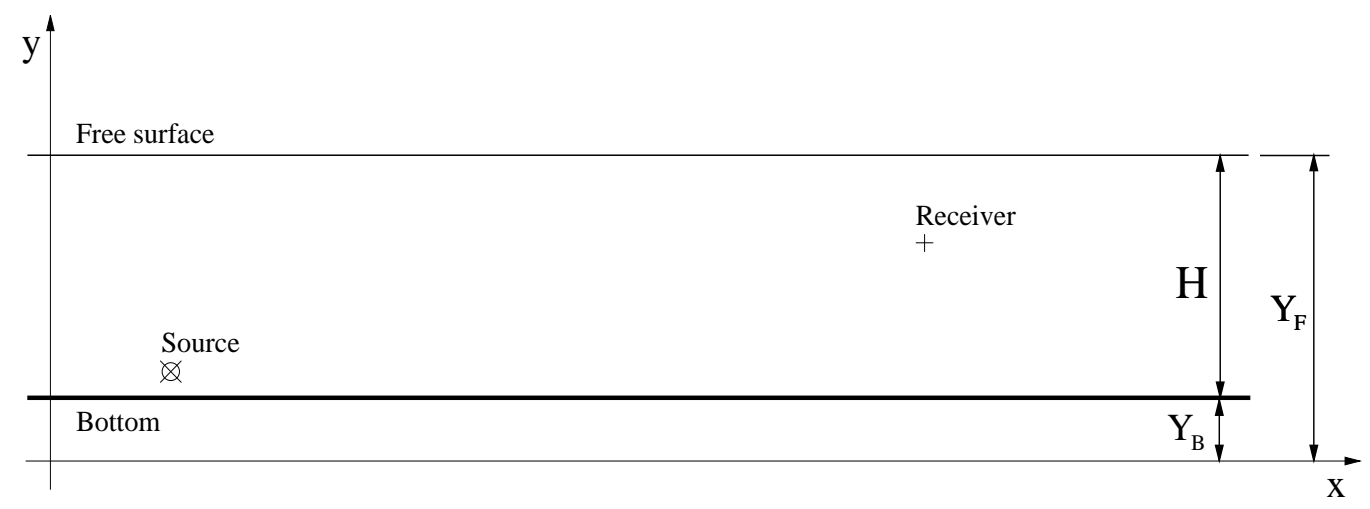

Figure 2: Geometry of the waveguide.

\subsubsection{Convergence tests}

Convergence tests were performed in order to assess the possible advantages and limitations of using Eqs. (4), (5) and (6) in either BEM or MFS models. The example refers to a waveguide with sound velocity $1500 \mathrm{~m} / \mathrm{s}$, subjected to a point source S applied at position $(0.00 \mathrm{~m}, 9.00 \mathrm{~m})$ as illustrated in Fig. 3. The responses were computed at receiver R1 placed at $(0.00 \mathrm{~m}, 5.00 \mathrm{~m})$ and at receiver $\mathrm{R} 2$ placed at $(5.00 \mathrm{~m}, 9.00 \mathrm{~m})$. The convergence test was performed for an excitation frequency of $1000 \mathrm{~Hz}$.

Figure 4 shows the responses for R1 and R2, respectively, where the amplitudes refer to the modulus of the acoustic pressure. From the analysis of Fig. 4(a) it was found that, as the receiver R1 is placed on the same vertical line of the source (S), a large number of terms is required for convergence of the series. On the other hand, as the receiver R2 is not on the 
same vertical line of the source, the number of terms required for convergence of the series is significantly reduced, as illustrated in Fig. 4(b).

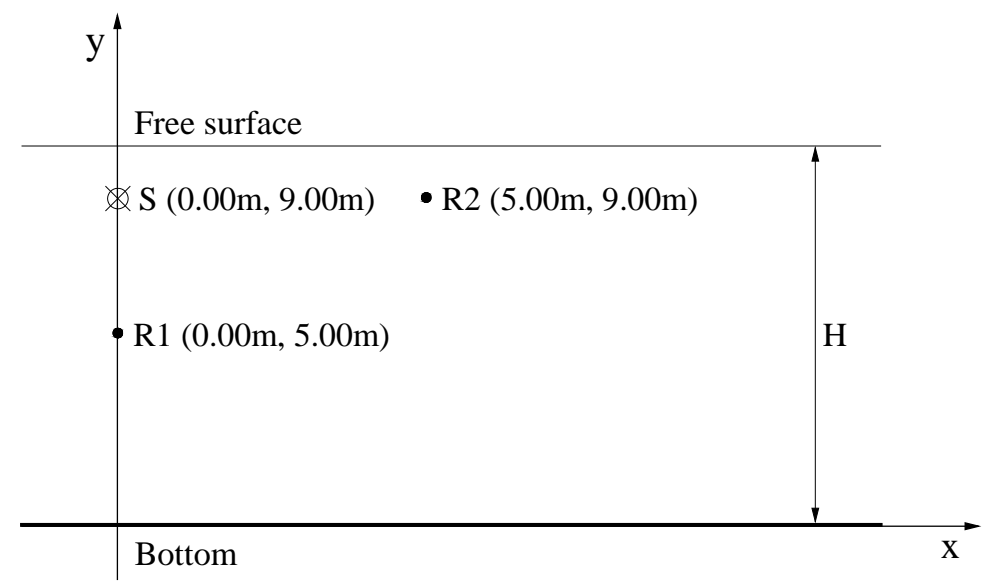

Figure 3: Geometry of the waveguide used in the convergence tests.

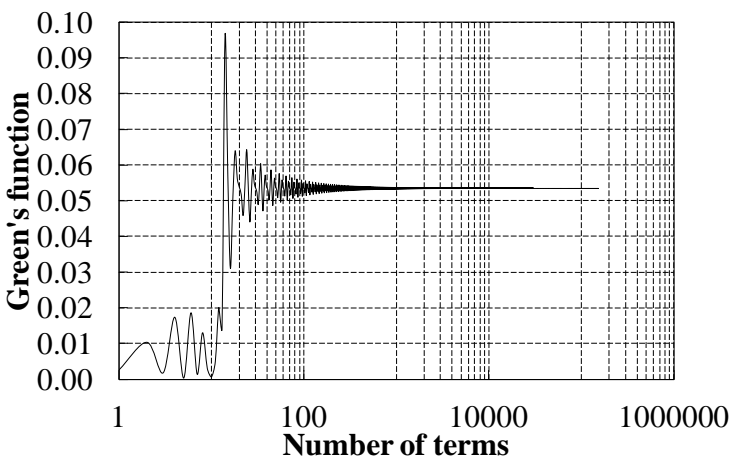

a)

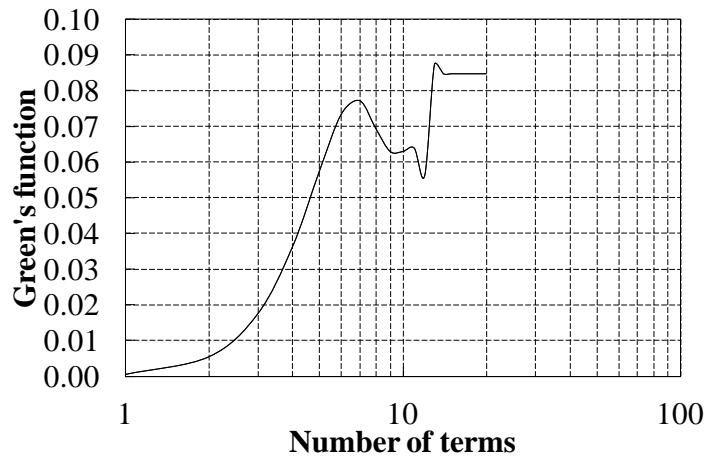

b)

Figure 4: Green's function responses as a function of the number of terms provided by source (S) for an excitation frequency of $1000 \mathrm{~Hz}$ : a) receiver $\mathrm{R} 1$ and b) receiver $\mathrm{R} 2$.

\subsection{Ewald's method}

Before applying Ewald's representation, the Green's function $G_{\mathrm{M}}(\boldsymbol{\xi}, \mathbf{x})$ given in Eq. (4) needs to be rewritten in the following form:

$G_{\mathrm{M}}(\boldsymbol{\xi}, \mathbf{x})=\frac{1}{H} \sum_{m=1}^{\infty} \sin \left[k_{y m}\left(Y_{\mathrm{F}}-y_{\xi}\right)\right] \sin \left[k_{y m}\left(Y_{\mathrm{F}}-y\right)\right] \frac{e^{-\sqrt{-\lambda_{m}}\left|x-x_{\xi}\right|}}{\sqrt{-\lambda_{m}}}$,

where $\lambda_{m}=k^{2}-k_{y m}^{2}$. 
After this procedure, the Green's function (7) can be defined by $G_{\mathrm{E}}(\boldsymbol{\xi}, \mathbf{x})$ as [13]:

$$
G_{\mathrm{E}}(\boldsymbol{\xi}, \mathbf{x})=\frac{2}{H} \sum_{m=1}^{\infty} \sin \left[k_{y m}\left(Y_{\mathrm{F}}-y_{\xi}\right)\right] \sin \left[k_{y m}\left(Y_{\mathrm{F}}-y\right)\right] \int_{0}^{\infty} \frac{e^{\lambda_{m} t-\left(\left(x-x_{\xi}\right)^{2} / 4 t\right)}}{\sqrt{4 \pi t}} d t
$$

Hence, when the source and field points are on the same vertical line, the final expression for Ewald's method can be written as:

$$
\begin{aligned}
G_{\mathrm{E}}(\boldsymbol{\xi}, \mathbf{x})= & \frac{1}{4 \pi} \sum_{n=0}^{\infty} C_{n}(\boldsymbol{\xi}, \mathbf{x})+\sum_{m=1}^{\infty} \frac{\cos (\pi m)}{4 \pi} \sum_{n=0}^{\infty} C_{m n}(\boldsymbol{\xi}, \mathbf{x})+ \\
& +\sum_{m=1}^{\infty} \frac{\sin \left[k_{y m}\left(Y_{\mathrm{F}}-y_{\xi}\right)\right] \sin \left[k_{y m}\left(Y_{\mathrm{F}}-y\right)\right] \operatorname{erfc}\left[b \sqrt{-\lambda_{m}}\right]}{H \sqrt{-\lambda_{m}}} .
\end{aligned}
$$

More details of the Ewald's representation can be found in Appendix A and in Santiago and Wrobel $[8,9]$.

\subsubsection{Convergence tests}

In order to verify the performance of the Green's function $G_{\mathrm{E}}(\boldsymbol{\xi}, \mathbf{x})$, a geometry was chosen which assumes two point sources and a line of receivers defined so that this problem allows undertanding the behaviour of the Green's function when implemented in BEM or MFS codes. This problem refers to a waveguide with constant depth of $10.00 \mathrm{~m}$ and subjected to a source S1 applied at position $(0.00 \mathrm{~m}, 1.00 \mathrm{~m})$ and to another source S2 applied at position $(0.00 \mathrm{~m}, 5.00 \mathrm{~m})$, as illustrated in Fig. 5 . The responses were computed along a line of receivers located on the same vertical alignment of the sources. Once again, the sound velocity and excitation frequency were taken to be $1500 \mathrm{~m} / \mathrm{s}$ and $1000 \mathrm{~Hz}$, respectively.

In the present paper, the constants $\alpha, \gamma$ and $\theta$ are assigned the empirical values 0.1 , 0.000001 and 20, respectively (see Appendix A). The corresponding functions $b(\beta)$ obtained with these values are shown in Fig. 6. 


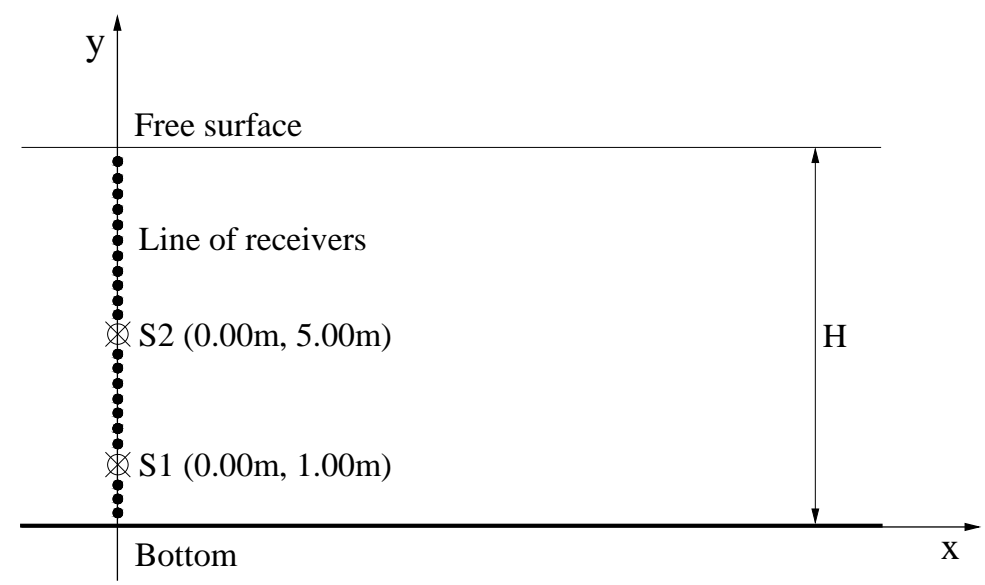

Figure 5: Geometry of the waveguide used in the convergence tests.

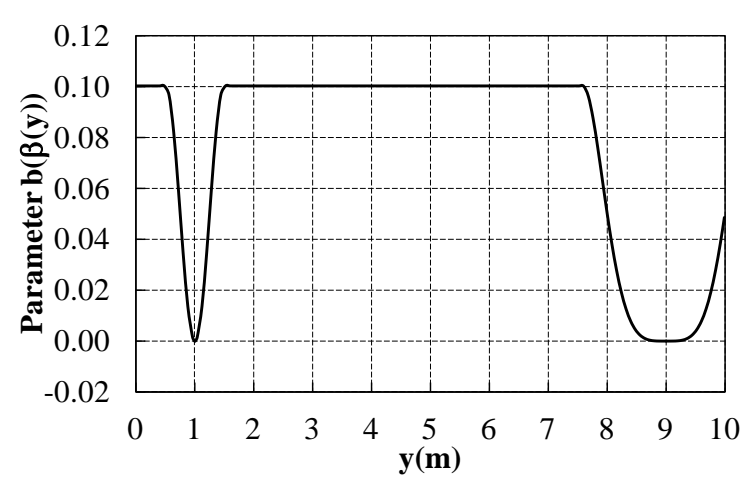

a)

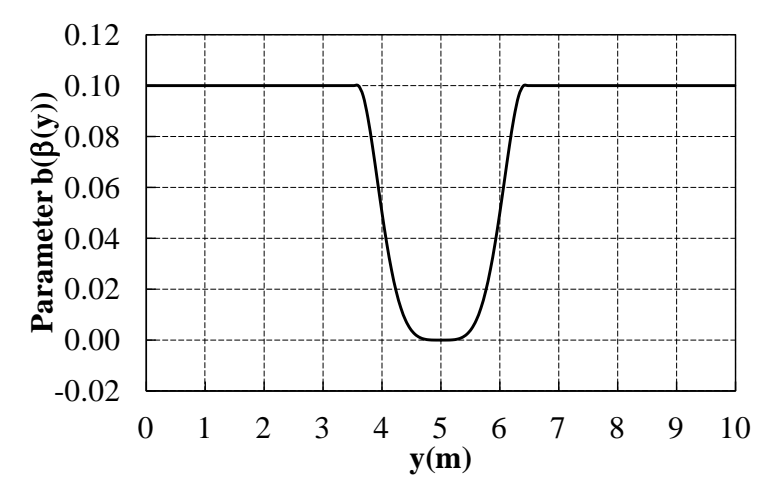

b)

Figure 6: Parameter $b$ for $H=Y_{\mathrm{F}}=10 \mathrm{~m}$ and $\left.Y_{\mathrm{B}}=0 \mathrm{~m}: \mathrm{a}\right)$ source $\left.\mathrm{S} 1 \mathrm{and} \mathrm{b}\right)$ source S2.

Figure 7 shows the verification of the functions $G_{\mathrm{M}}(\xi, \mathbf{x})$ and $G_{\mathrm{E}}(\xi, \mathbf{x})$ using the function $b(\beta)$ for an excitation frequency of $1000 \mathrm{~Hz}$, for the sources S1 and S2, respectively. The analysis of these results clearly confirms that there is an excellent agreement between the two Green's functions, confirming the validity of Ewald's representation. In Fig. 8, curves representing the convergence behaviour of the Ewald's method are shown. The curves clearly reveal a small relative error (below $10^{-2}$ ) of the proposed method compared to the eigenfunction expansion solution.

From the analysis of Fig. 9, which plots the number of terms required for both Green's functions to achieve convergence, it can be seen that a much faster series is produced by the function $G_{\mathrm{E}}(\boldsymbol{\xi}, \mathbf{x})$. 
Real part

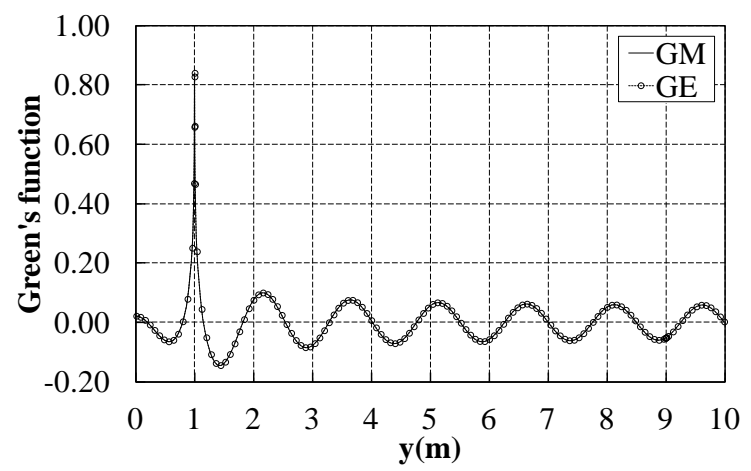

a1)

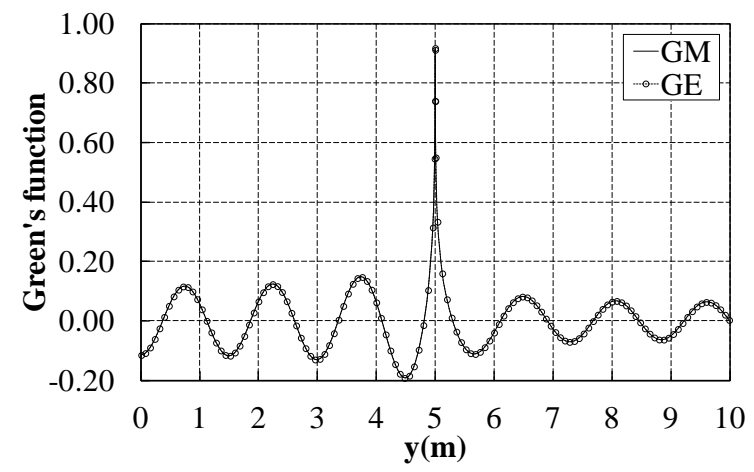

b1)
Imaginary part

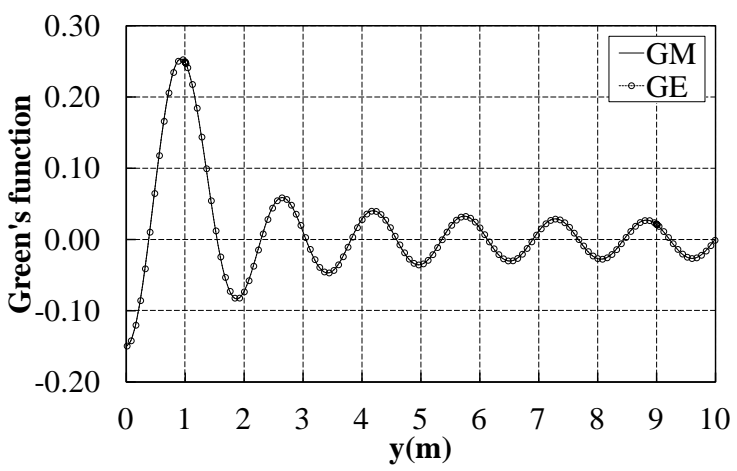

a2)

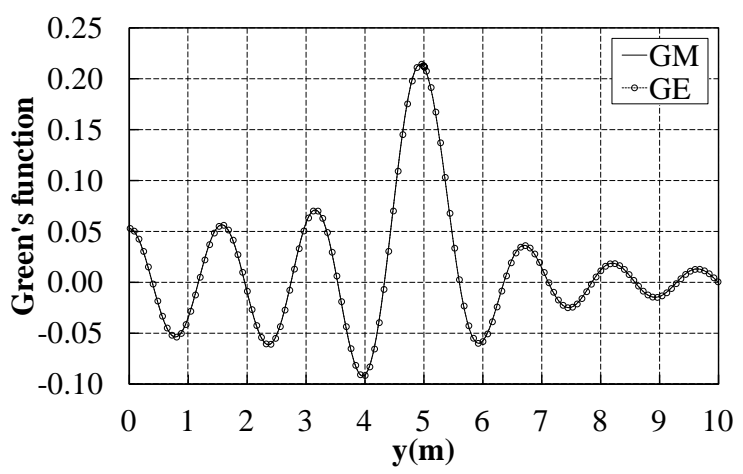

b2)

Figure 7: Verification of the Green's functions $G_{\mathrm{M}}(\boldsymbol{\xi}, \mathbf{x})$ and $G_{\mathrm{E}}(\boldsymbol{\xi}, \mathbf{x})$ for an excitation frequency of $1000 \mathrm{~Hz}$ : a) source S1 and b) source S2.

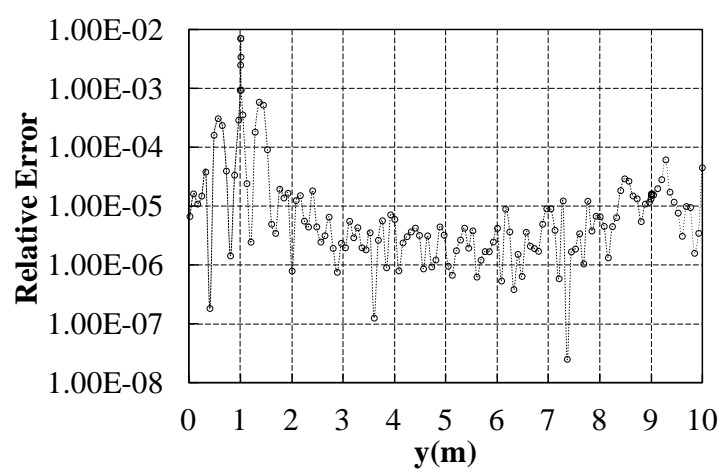

a)

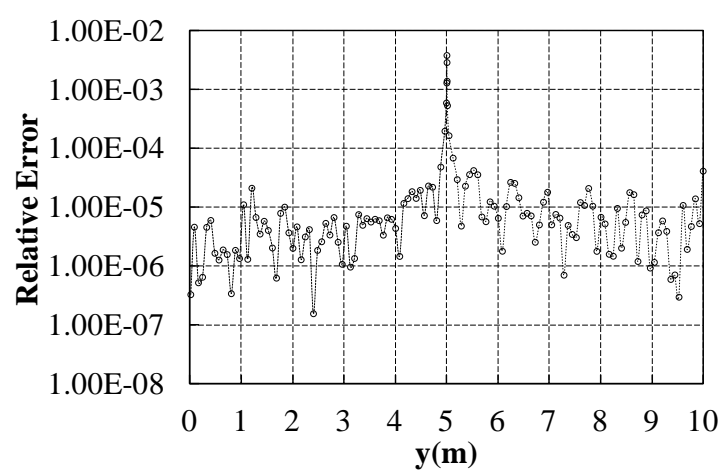

b)

Figure 8: Convergence of the Green's function $G_{\mathrm{E}}(\boldsymbol{\xi}, \mathbf{x})$ for an excitation frequency of $1000 \mathrm{~Hz}$ : a) source S1 and b) source S2. 


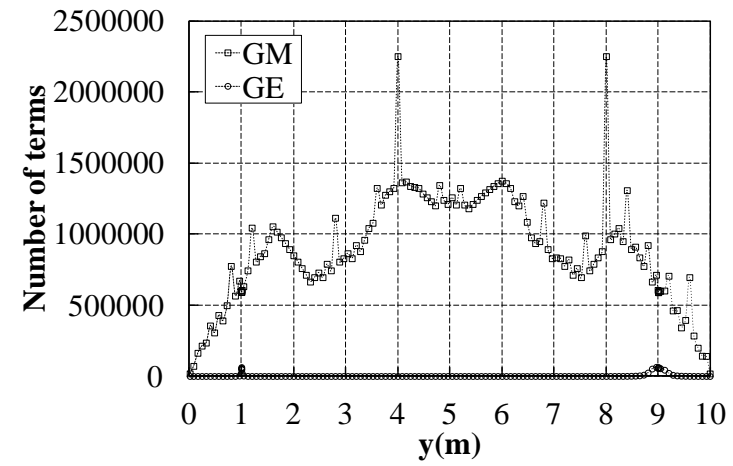

a)

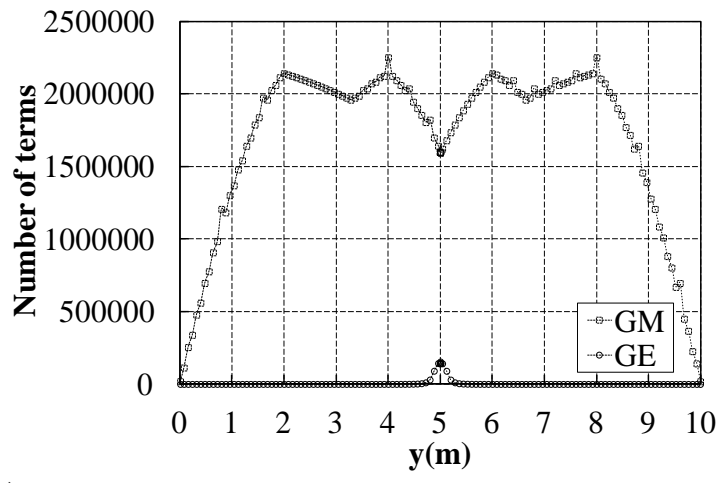

b)

Figure 9: Number of terms of the Green's functions $G_{M}(\boldsymbol{\xi}, \mathbf{x})$ and $G_{\mathrm{E}}(\boldsymbol{\xi}, \mathbf{x})$ for an excitation frequency of $1000 \mathrm{~Hz}$ : a) source S1 and b) source S2.

\subsubsection{Implementation of the functions in the numerical models}

It is important to remember that the implementation of these Green's functions in the BEM models requires the calculation of singular integrals when the functional and nodal points coincide. To eliminate this singularity, the free space Green's function is subtracted and added to the Green's function in Eqs. (7) and (9), in the form:

$$
\int_{\Gamma} G(\boldsymbol{\xi}, \mathbf{x}) d \Gamma=\int_{-1}^{1}\left[G_{\mathrm{M}, \mathrm{E}}(\boldsymbol{\xi}, \mathbf{x})-\frac{\mathrm{i}}{4} H_{0}^{(1)}(k r)\right]|J| d \eta+\int_{-1}^{1}\left[\frac{\mathrm{i}}{4} H_{0}^{(1)}(k r)\right]|J| d \eta,
$$

where $H_{0}^{(1)}$ is the Hankel function of the first kind of order zero and $|J|$ is the Jacobian. It is important to note that $\frac{\mathrm{i}}{4} H_{0}^{(1)}(k r)$ refers to the full-space Green's function. The resulting integrals in Eq. (10) can then be computed in two parts: the first term is integrated numerically using standard Gauss quadrature, since the singularity has been removed; the second term can be integrated analytically, following Tadeu et al. [18, 19].

\section{NUMERICAL FORMULATIONS}

The previously described two-dimensional acoustic wave propagation problem is solved using three efficient numerical models in the frequency domain, based on the BEM and on the MFS. 


\subsection{Boundary Element Method}

According to Green's second identity, the following classical boundary integral equation can be obtained:

$C(\boldsymbol{\xi}) p(\boldsymbol{\xi})=-\mathrm{i} \omega \rho \int_{\Gamma} G(\boldsymbol{\xi}, \mathbf{x}) \nu_{n}(\mathbf{x}) d \Gamma-\int_{\Gamma} \frac{\partial G(\boldsymbol{\xi}, \mathbf{x})}{\partial \mathbf{n}} p(\mathbf{x}) d \Gamma+Q G\left(\xi^{s}, \boldsymbol{\xi}\right)$

where $\Gamma$ is equal to $\Gamma_{\mathrm{F}} \cup \Gamma_{\mathrm{B}} ; G(\xi, \mathbf{x})$ is the Green's functions; $p(\mathbf{x})$ and $v_{n}(\mathbf{x})$ represent the acoustic pressure and the normal derivative of the acoustic pressure, respectively. The coefficient $C(\xi)$ depends on the boundary geometry at the source point $\xi$. This coefficient takes the value 0.5 if the boundary is smooth and 1.0 at any point within the domain.

In order to solve Eq. (11), the boundary integral equation requires the discretization of all boundaries, if special Green's functions are not used. However, the problem will be solved herein by using the previously described BEM formulation which adopts Green's functions that satisfy specific boundary conditions, allowing reducing the discretization of the proposed models. The formulation refers to a specific shallow water configuration, taking into account the presence of a step up on the rigid bottom and of a free surface, as defined in Fig. 10, excited by a point source.

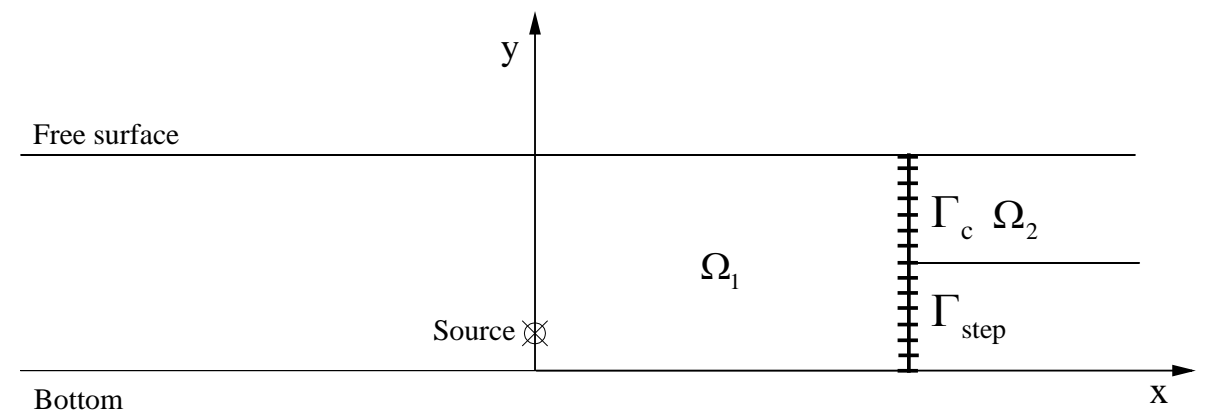

a)

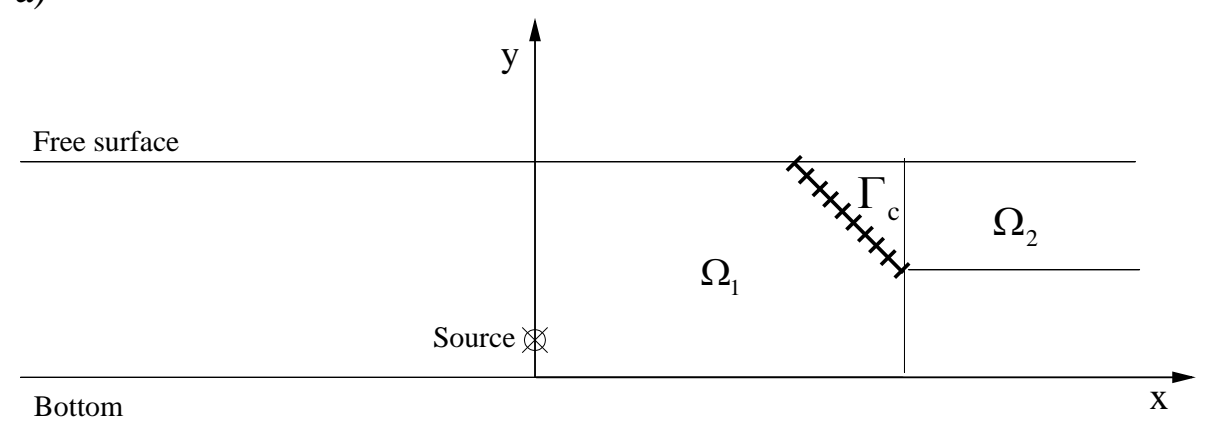

b)

Figure 10: Geometry of the BEM models: a) Model 1 and b) Model 2. 
Two different models are proposed here: the first one (Model 1) makes use of the subregion technique with a Green's function designated as $G_{\mathrm{E}}(\boldsymbol{\xi}, \mathbf{x})$ for each sub-region, requiring only the discretization of the vertical interface $\Gamma_{c}$ (see Fig. 10(a)). Hence, introducing the appropriate conditions along the common vertical interface $\Gamma_{\mathrm{c}}$ (where continuity of pressure and velocity is imposed), and the Neumann condition $\left(v_{n}(\mathbf{x})=0\right)$ in the boundary of the step designated as $\Gamma_{\text {step }}$, and considering a total of $N N$ functional nodes with constant elements ( $N N=N E_{\mathrm{s}}+N E_{\mathrm{c}}$ ), the following equations can be obtained:

$$
\begin{aligned}
C\left(\xi_{i}\right) p\left(\xi_{i}\right)= & -\mathrm{i} \omega \rho \sum_{j=N E_{s}+1}^{N E_{s}+N E_{c}} \int_{\Gamma_{j}} G_{\mathrm{E}}^{\mathrm{H}_{1}}\left(\boldsymbol{\xi}_{i}, \mathbf{x}_{j}\right) v_{n}\left(\mathbf{x}_{j}\right) d \Gamma-\sum_{j=1}^{N E_{s}+N E_{c}} \int_{\Gamma_{j}} \frac{\partial G_{\mathrm{E}}^{\mathrm{H}_{1}}\left(\boldsymbol{\xi}_{i}, \mathbf{x}_{j}\right)}{\partial \mathbf{n}} p\left(\mathbf{x}_{j}\right) d \Gamma+ \\
& +(1-Q) G_{\mathrm{E}}^{\mathrm{H}_{1}}\left(\xi^{s}, \boldsymbol{\xi}_{i}\right) ; \quad i=1, \ldots, N N \quad \text { region } \Omega_{1} \\
C\left(\xi_{i}\right) p\left(\xi_{i}\right)= & -\mathrm{i} \omega \rho \sum_{j=N E_{s}+1}^{N E_{\mathrm{s}}+N E_{c}} \int_{\Gamma_{j}} G_{\mathrm{E}}^{\mathrm{H}_{2}}\left(\boldsymbol{\xi}_{i}, \mathbf{x}_{j}\right) \nu_{n}\left(\mathbf{x}_{j}\right) d \Gamma-\sum_{j=N E_{s}+1}^{N E_{s}+N E_{c}} \int_{\Gamma_{j}} \frac{\partial G_{\mathrm{E}}^{\mathrm{H}_{2}}\left(\boldsymbol{\xi}_{i}, \mathbf{x}_{j}\right)}{\partial \mathbf{n}} p\left(\mathbf{x}_{j}\right) d \Gamma+ \\
& +Q G_{\mathrm{E}}^{\mathrm{H}_{2}}\left(\xi^{s}, \boldsymbol{\xi}_{i}\right) ; \quad i=N E_{s}+1, \ldots, N N \quad \text { region } \Omega_{2},
\end{aligned}
$$

where $G_{\mathrm{E}}^{\mathrm{H}_{1}}\left(\boldsymbol{\xi}_{i}, \mathbf{x}_{j}\right)$ and $G_{\mathrm{E}}^{\mathrm{H}_{2}}\left(\boldsymbol{\xi}_{i}, \mathbf{x}_{j}\right)$ are the Green's functions for a waveguide with flat rigid bottom and free surface at the boundary element $\mathbf{x}_{j}$, whose details were given in a previous section; $G_{\mathrm{E}}^{\mathrm{H}_{1}}\left(\xi^{s}, \xi_{i}\right)$ and $G_{\mathrm{E}}^{\mathrm{H}_{2}}\left(\xi^{s}, \xi_{i}\right)$ are the incident fields regarding the acoustic pressure by the point source placed at position $\xi^{s} ; Q=1$ if the source is positioned in region $\Omega_{2}$ while $Q=0$ if the source is positioned in region $\Omega_{1}$. The notation $H_{1}$ refers to the higher depth of the channel and the notation $\mathrm{H}_{2}$ refers to the lower depth of the channel. The velocity $v_{n}$ is the particle velocity along the normal direction and $\mathbf{n}$ is the unit normal vector pointing outward of each sub-region (i.e., with opposing directions for the two sub-regions).

Considering that $N E_{s}$ elements are used to discretize the boundary of the step and $N E_{c}$ elements are used to discretize the common interface between the sub-regions, a system of $N E_{s}+2 N E_{c}$ equations on $N E_{s}+2 N E_{c}$ unknown values may be written. Solving this system of equations makes it possible to obtain the acoustic pressure at any domain point through the boundary integral equation for internal points. 
The second model (Model 2) makes use of a Green's function designated as $G_{\mathrm{M}}^{\mathrm{H}_{2}}\left(\boldsymbol{\xi}_{i}, \mathbf{x}_{j}\right)$ in the region $\Omega_{2}$ that directly satisfies the boundary conditions at the flat rigid bottom and flat free surface, while for region $\Omega_{1}$ the Green's function also takes into account the presence of the step by applying the image-source method, designated as $G_{\mathrm{M}}^{\text {step }}\left(\boldsymbol{\xi}_{i}, \mathbf{x}_{j}\right)$. A sub-region technique is again applied by defining two sub-regions, with only the inclined interface inbetween them requiring discretization (see Fig. 10(b)). Therefore, introducing the appropriate conditions in the inclined interface, the following equations can be written:

$$
\begin{aligned}
C\left(\xi_{i}\right) p\left(\xi_{i}\right)= & -\mathrm{i} \omega \rho \sum_{j=1}^{N E} \int_{\Gamma_{j}} G_{\mathrm{M}}^{\text {step }}\left(\boldsymbol{\xi}_{i}, \mathbf{x}_{j}\right) v_{n}\left(\mathbf{x}_{j}\right) d \Gamma-\sum_{j=1}^{N E} \int_{\Gamma_{j}} \frac{\partial G_{\mathrm{M}}^{\text {step }}\left(\boldsymbol{\xi}_{i}, \mathbf{x}_{j}\right)}{\partial \mathbf{n}} p\left(\mathbf{x}_{j}\right) d \Gamma+ \\
& +(1-Q) G_{\mathrm{M}}^{\text {step }}\left(\xi^{s}, \boldsymbol{\xi}_{i}\right) ; \quad i=1, \ldots, N N \quad \text { region } \Omega_{1} \\
C\left(\xi_{i}\right) p\left(\xi_{i}\right)= & -\mathrm{i} \omega \rho \sum_{j=1}^{N E} \int_{\Gamma_{j}} G_{\mathrm{M}}^{\mathrm{H}_{2}}\left(\boldsymbol{\xi}_{i}, \mathbf{x}_{j}\right) v_{n}\left(\mathbf{x}_{j}\right) d \Gamma-\sum_{j=1}^{N E} \int_{\Gamma_{j}} \frac{\partial G_{\mathrm{M}}^{\mathrm{H}_{2}}\left(\boldsymbol{\xi}_{i}, \mathbf{x}_{j}\right)}{\partial \mathbf{n}} p\left(\mathbf{x}_{j}\right) d \Gamma+ \\
& +Q G_{\mathrm{M}}^{\mathrm{H}_{2}}\left(\xi^{s}, \boldsymbol{\xi}_{i}\right) ; \quad i=1, \ldots, N N \quad \text { region } \Omega_{2}
\end{aligned}
$$

where $N E=N E_{c}=N N$ (constant elements).

Solving the linear system of $2 N E$ equations on $2 N E$ unknowns makes it possible to obtain the nodal values of the acoustic pressure and normal component of the velocity in the inclined interface $\Gamma_{c}$. Thus, the acoustic pressure can be obtained at any point of the acoustic domain through the boundary integral equation for internal points.

\subsection{Method of Fundamental Solutions}

In this sub-section, a single proposed model was defined using the MFS, as schematically illustrated in Fig. 11. This model makes use of the sub-regions technique, with the Green's function designated as $G_{\mathrm{M}}(\boldsymbol{\xi}, \mathbf{x})$ applied for each sub-region, requiring only the discretization of the vertical interface $\Gamma_{\mathrm{c}}$ (as in Model 1). In both regions, the Green's function that directly satisfies the rigid bottom and free flat surface is assumed. Thus, imposing continuity of the acoustic pressure and normal component of the velocity at each interface point $\mathbf{x}$, the following equations can be obtained: 


$$
\begin{aligned}
& \sum_{n=1}^{N V S_{c}+N V S_{s}} \mathrm{~A}_{n} G_{\mathrm{M}}^{\mathrm{H}_{1}}\left(\boldsymbol{\xi}_{n}, \mathbf{x}\right)+(1-Q) G_{\mathrm{M}}^{\mathrm{H}_{1}}\left(\xi^{s}, \mathbf{x}\right)=\sum_{n=1}^{N V S_{c}} \mathrm{~B}_{n} G_{\mathrm{M}}^{\mathrm{H}_{2}}\left(\boldsymbol{\xi}_{n}, \mathbf{x}\right)+Q G_{\mathrm{M}}^{\mathrm{H}_{2}}\left(\xi^{s}, \mathbf{x}\right), \\
& \sum_{n=1}^{N V S_{\mathrm{c}}+N V S_{s}} \mathrm{~A}_{n} \frac{\partial G_{\mathrm{M}}^{\mathrm{H}_{1}}\left(\boldsymbol{\xi}_{n}, \mathbf{x}\right)}{\partial \mathbf{n}_{1}}+(1-Q) \frac{\partial G_{\mathrm{M}}^{\mathrm{H}_{1}}\left(\boldsymbol{\xi}^{s}, \mathbf{x}\right)}{\partial \mathbf{n}}=\sum_{n=1}^{N F V_{c}} \mathrm{~B}_{n} \frac{\partial G_{\mathrm{M}}^{\mathrm{H}_{2}}\left(\boldsymbol{\xi}_{n}, \mathbf{x}\right)}{\partial \mathbf{n}}+Q \frac{\partial G_{\mathrm{M}}^{\mathrm{H}_{2}}\left(\xi^{s}, \mathbf{x}\right)}{\partial \mathbf{n}},
\end{aligned}
$$

where $\mathrm{A}_{n}$ and $\mathrm{B}_{n}$ are the amplitudes to be determined for each virtual source; $Q=1$ if the real source is positioned in region $\Omega_{2}$ and $Q=0$ if the real source is located in region $\Omega_{1}$; $G_{\mathrm{M}}^{\mathrm{H}_{1}}\left(\xi^{s}, \mathbf{x}\right)$ and $G_{\mathrm{M}}^{\mathrm{H}_{2}}\left(\xi^{s}, \mathbf{x}\right)$ are the incident fields regarding the acoustic pressure generated by the real source; $N V S_{\text {c }}$ corresponds to the number of virtual sources placed at each sub-region and $N V S_{s}$ corresponds to the number of virtual sources positioned in region $\Omega_{1} ; G_{\mathrm{M}}^{\mathrm{H}_{1}}\left(\boldsymbol{\xi}_{n}, \mathbf{x}\right)$ and $G_{\mathrm{M}}^{\mathrm{H}_{2}}\left(\boldsymbol{\xi}_{n}, \mathbf{x}\right)$ refer to the Green's functions for a flat rigid bottom and flat free surface, whose details were given in the previous section.

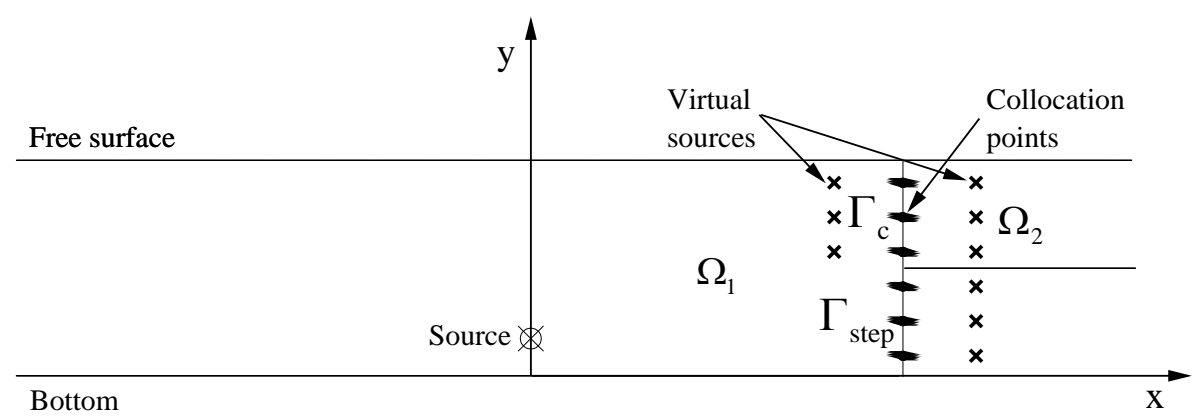

Figure 11: Geometry of the MFS model.

Imposing the Neumann condition $\left(v_{n}(\mathbf{x})=0\right)$ at each collocation point $\mathbf{x}$ of the vertical boundary $\Gamma_{\text {step }}$, the following equation can be written:

$$
\sum_{n=1}^{N V S_{s}+N V S_{c}} \mathrm{~A}_{n} \frac{\partial G_{\mathrm{M}}^{\mathrm{H}_{1}}\left(\boldsymbol{\xi}_{n}, \mathbf{x}\right)}{\partial \mathbf{n}}=-(1-Q) \frac{\partial G_{\mathrm{M}}^{\mathrm{H}_{1}}\left(\xi^{s}, \mathbf{x}\right)}{\partial \mathbf{n}}
$$

Therefore, a linear system of $N V S_{s}+2 N V S_{c}$ equations on $N V S_{s}+2 N V S_{c}$ unknowns may be written. Once this system of equations is solved for the relevant unknown amplitudes, the response at any point of the domain may be obtained by using the following equations: 


$$
\begin{aligned}
& \sum_{n=1}^{N V S_{c}+N V S_{s}} \mathrm{~A}_{n} G_{\mathrm{M}}^{\mathrm{H}_{1}}\left(\xi_{n}, \mathbf{x}\right)+(1-Q) G_{\mathrm{M}}^{\mathrm{H}_{1}}\left(\xi^{s}, \mathbf{x}\right) \text { region } \Omega_{1}, \\
& \sum_{n=1}^{N V S_{c}} \mathrm{~B}_{n} G_{\mathrm{M}}^{\mathrm{H}_{2}}\left(\xi_{n}, \mathbf{x}\right)+Q G_{\mathrm{M}}^{\mathrm{H}_{2}}\left(\xi^{s}, \mathbf{x}\right) \text { region } \Omega_{2} .
\end{aligned}
$$

\section{BEHAVIOUR OF THE BEM AND MFS MODELS}

In order to verify and analyse the behaviour of the proposed BEM and MFS models used in this work, the described formulations were implemented and a test case was analysed. We consider the geometry depicted in Fig. 12(a), consisting of a flat waveguide containing a step on the bottom of the sea. For this configuration, the response is generated by a point source positioned at $5.0 \mathrm{~m}$ depth and computed at one horizontal line of receivers, placed at depth of $2.5 \mathrm{~m}$. The acoustic medium is assumed to be water, with density of $1000 \mathrm{~kg} / \mathrm{m}^{3}$ and a sound propagation velocity of $1500 \mathrm{~m} / \mathrm{s}$.

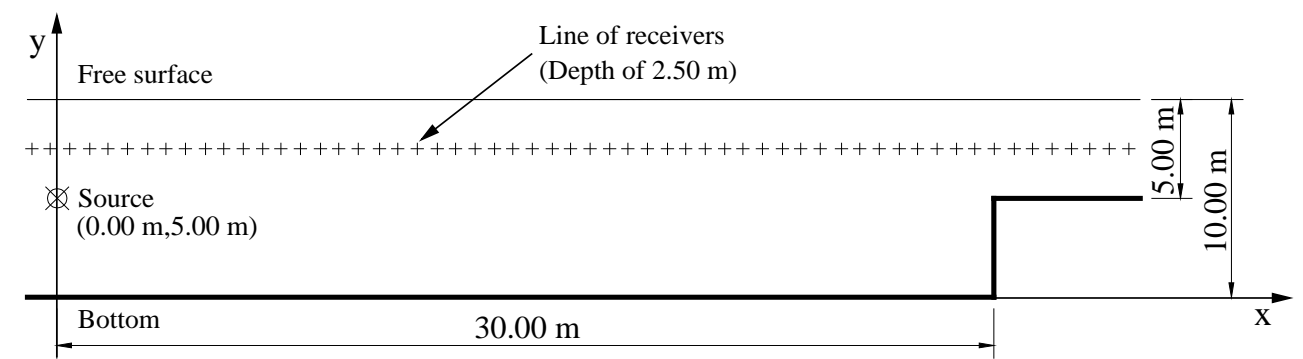

a)

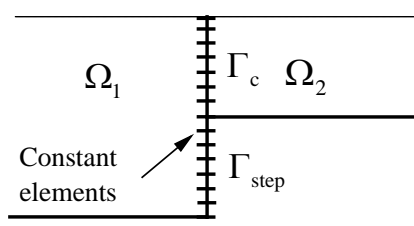

b)

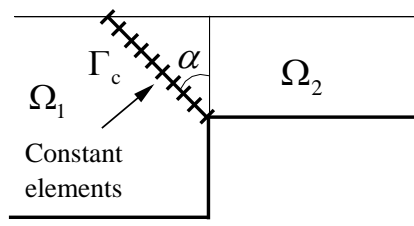

c)

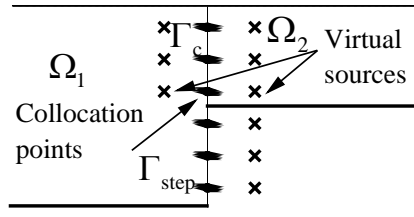

d)

Figure 12: a) Geometry of the problem, b) Model 1: geometry with discretized vertical interface (BEM), c) Model 2: geometry with discretized inclined interface (BEM) and d) MFS Model: geometry and position of collocation points and fictitious sources. 
The models require the discretization of either the vertical interface between the subregions of different depth of the waveguide (Model 1) or an inclined interface with an angle of $\alpha=2.3^{\circ}$ to the left side (Model 2), as illustrated in Figs. 12(b) and (c), respectively. The parameters $\alpha=0.1, \gamma=0.000001$ and $\theta=20$ (empirical values) for function $b(\beta)$ were adopted in the BEM analysis of Model 1. To build the MFS model, boundary points are located over a vertical interface and two sets of virtual source points may be defined (see Fig. 12(d)). In this model, a distance between the vertical interface and the virtual source points equal to $1.0 \mathrm{~m}$ was adopted.

When using the BEM models the number of boundary elements is defined as a function of the frequency, by using a relation between the incident wavelength and the length of the boundary elements equal to a minimum of 10. For the MFS model, the number of collocation points was also defined as a function of the frequency and computed using a relation of 5 between the incident wavelength and the distance between collocation points.

Figure 13 illustrates the response computed for frequencies of $125 \mathrm{~Hz}, 250 \mathrm{~Hz}, 500 \mathrm{~Hz}$ and $1000 \mathrm{~Hz}$ using the three methods, for a set of receivers located at a depth of $2.50 \mathrm{~m}$. It is clear that an excellent agreement occurs between all three models, with accurate responses being computed by all of them. It should be mentioned that the three approaches were found to be numerically efficient, and allowed overcoming the Green's function's convergence problems that occur when using the BEM together with the classic eigenfunction expansion, given by eq. (7).

\subsection{Comparison of the three numerical models}

The BEM model 1 makes use of Green's functions based on the Ewald's Method for each sub-region and the sub-region technique is employed, requiring only the discretization of a vertical interface. The Ewald's method is used here in order to improve the convergence of the Green's functions in terms of normal modes, particularly when the source and field points are located on the same vertical alignment, significantly improving the performance of the BEM. 
Real part

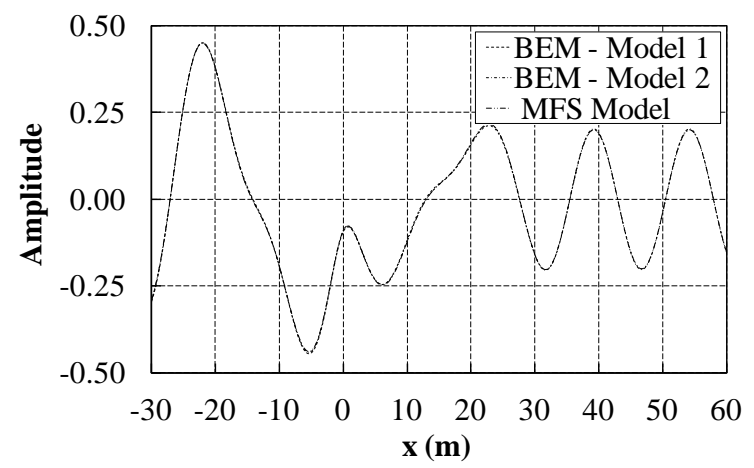

a1)

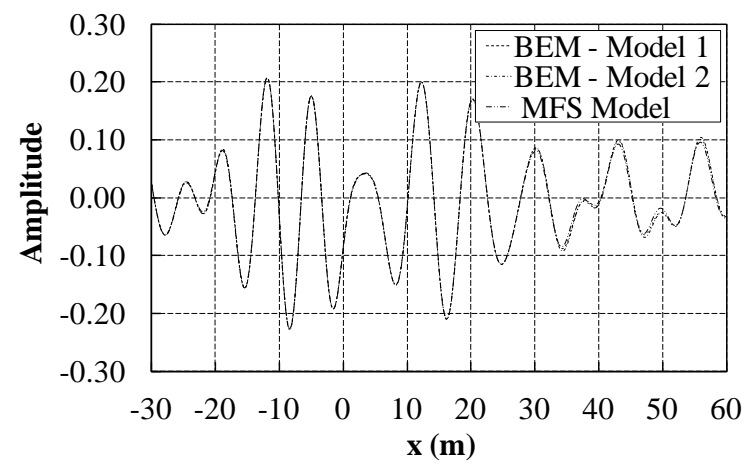

b1)

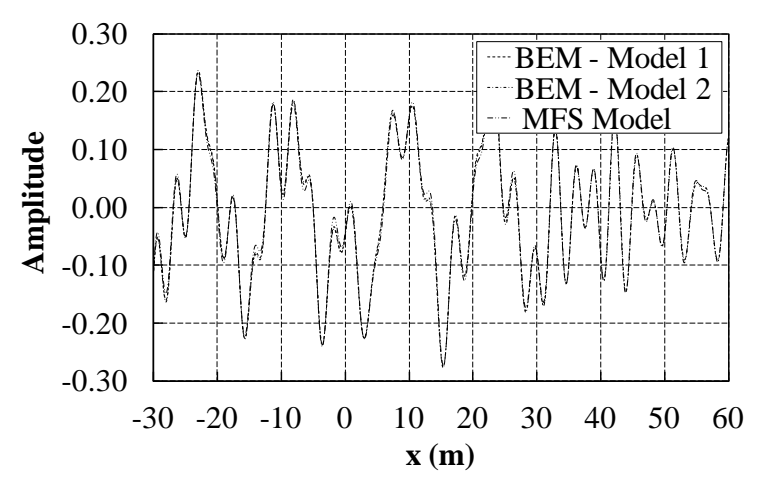

c1)

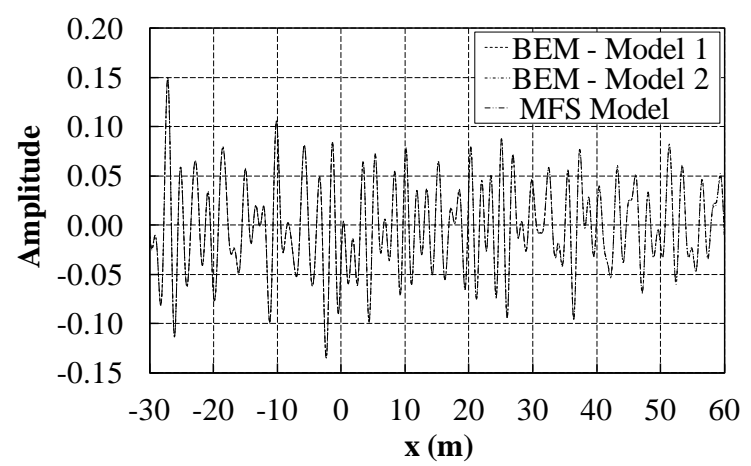

d1)
Imaginary part

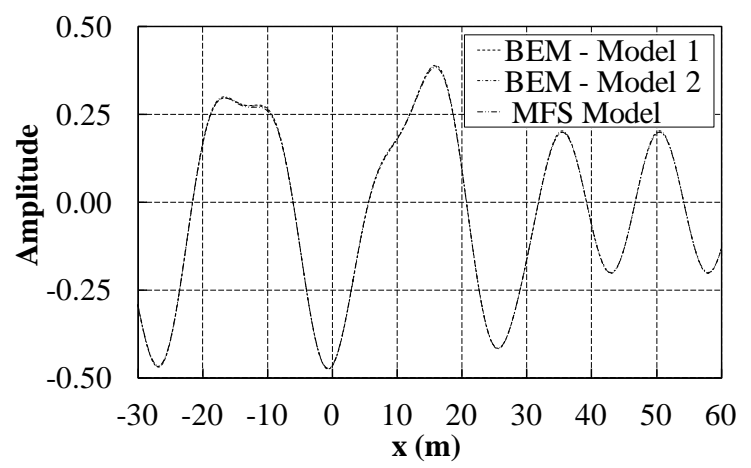

a2)

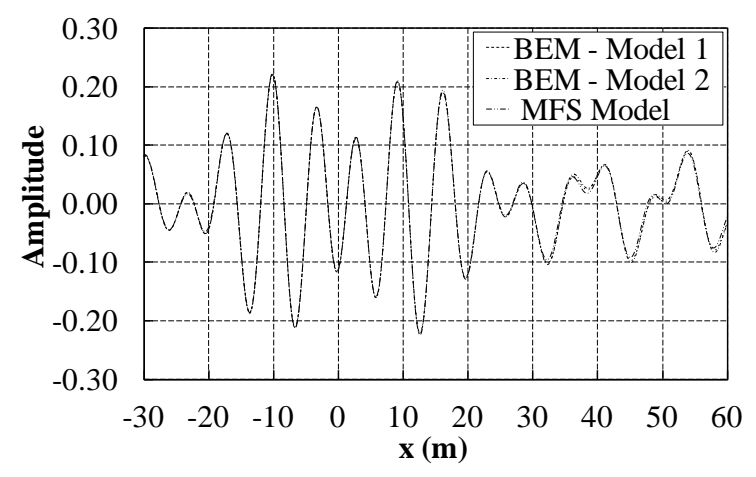

b2)

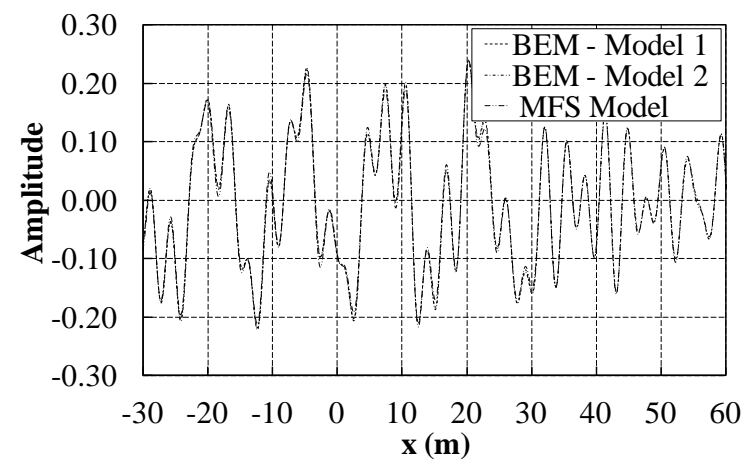

c2)

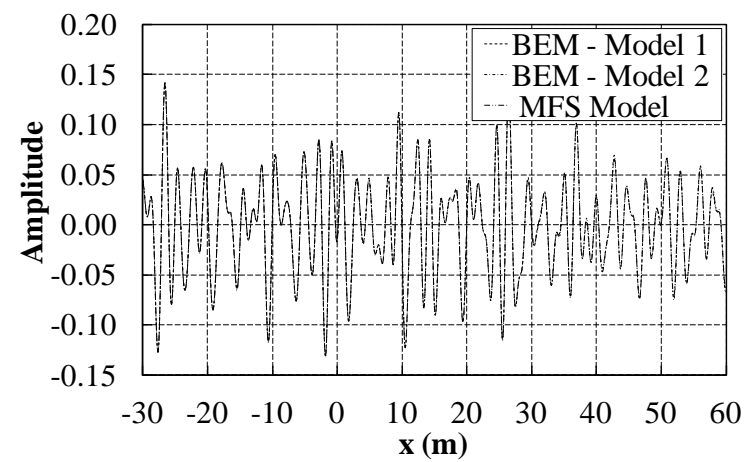

d2)

Figure 13. Response calculated at one horizontal line of receivers placed at depth of $2.5 \mathrm{~m}$ for the three numerical models: a) $125 \mathrm{~Hz}$, b) $250 \mathrm{~Hz}$, c) $500 \mathrm{~Hz}$ and d) $1000 \mathrm{~Hz}$. 
The BEM model 2 and the MFS model make use of Green's functions based on the normal mode solution for each sub-region and the sub-region technique is again applied. Note that the convergence problem of the series in terms of normal modes occurs when the source and the receiver are positioned on the same vertical line, as the exponential term of the eigenfunction expansion is equal to one in this case $\left(x=x_{\xi}\right)$. In model 2, this problem is overcome by inclining the interface between the two sub-regions within the waveguide. Therefore, the convergence of these Green's functions is fast and occurs with a small number of terms. In the MFS model, this convergence problem is also overcome because the virtual sources required to construct the response are never in the same vertical line of the collocation points, allowing a much faster convergence of the Green’s functions.

The three numerical models were found to be numerically efficient, but it should be mentioned that the number of collocation points used to calculate the MFS results is two times smaller than the number of boundary elements used in the BEM models. Moreover, the MFS does not require the numerical and analytical integrations needed in the BEM models. Therefore, the MFS model was found to be the most efficient of the three proposed numerical approaches to study underwater acoustic wave propagation in the frequency domain.

\section{NUMERICAL EXAMPLES}

To illustrate the applicability of the proposed formulations, the problem shown in Fig. 14 is analysed in this section using only the MFS model. In underwater acoustics, it is common to use the Transmission Loss ( $\mathrm{TL}=-10 \log \left(p^{2} / p_{0}^{2}\right)$ with $p_{0}$ being the pressure of the incident field generated by a point source at a distance of $1.00 \mathrm{~m}$ ) to calculate the response of a given underwater system. It is thus important to assess the influence of the step on the bottom of the sea using the TL. Once again, the acoustic medium is assumed to be water, with density of $1000 \mathrm{~kg} / \mathrm{m}^{3}$ and sound propagation velocity of $1500 \mathrm{~m} / \mathrm{s}$.

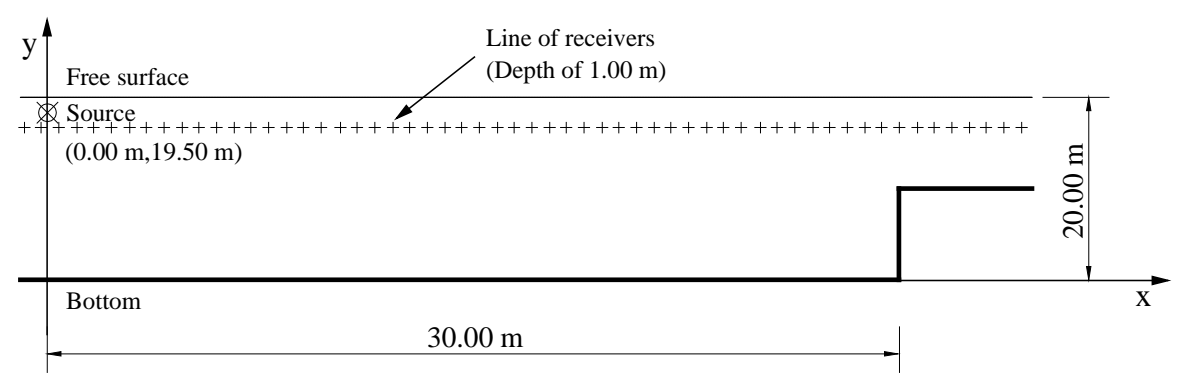

Figure 14: Geometry of the numerical example. 
Figure 15 displays the TL plots computed along the line of receivers placed $1.00 \mathrm{~m}$ below the free surface of the sea when an excitation source is at position $(0.00 \mathrm{~m}, 19.50 \mathrm{~m})$. In these plots, the dashed curve refers to the reference case of a waveguide with free surface and flat rigid bottom, while the continuous curve refers to the configurations of the proposed MFS model. A vertical dashed line is also included to mark the transition between the two sub-regions of different depth of the waveguide. The transmission loss is computed for four different excitation frequencies, so that the response includes the contribution of 1 ( $50 \mathrm{~Hz}), 3$ $(100 \mathrm{~Hz}), 5(250 \mathrm{~Hz})$ and $13(500 \mathrm{~Hz})$ propagating modes of the waveguide with constant depth of $20.0 \mathrm{~m}$.

For all frequencies, the TL responses of the waveguide containing a step up present significant differences in relation to the TL responses considering only a horizontal waveguide. It can be seen that the TL curves in this waveguide exhibit only few oscillations along the computed receivers for frequencies of $50 \mathrm{~Hz}$ and $100 \mathrm{~Hz}$, whereas for the frequencies of $250 \mathrm{~Hz}$ and $500 \mathrm{~Hz}$ the TL curves show a sequence of peaks corresponding to a large number of propagating modes within the system, particularly at the frequency of $500 \mathrm{~Hz}$. However, when the waveguide containing a step up is considered, a pronounced interference occurs between the source and the step up related to the waves that are reflected by the step, particularly at the higher frequencies ( $250 \mathrm{~Hz}$ and $500 \mathrm{~Hz}$ ). Therefore, the values computed for the TL tend to be closer to those responses calculated for the waveguide (see Figs. 15(c) and (d)). For this reason, the TL plots are more difficult to be analysed at the higher frequencies due to the pronounced interference between the reflections that occur within this system due to presence of the step up. It is important to note that the lower frequencies produce less propagating modes with higher amplitudes, while the higher frequencies allow a large number of propagating modes.

For the lower frequencies ( $50 \mathrm{~Hz}$ and $100 \mathrm{~Hz}$ ), the receptors further away from the source have a constant TL, indicating that there are few propagating modes (see Figs. 15(a1), (b1) and (b2)), while Fig. 15(a2) displays a different characteristic, with an exponential increase of the TL when the wave travels farther away from the source. This behaviour indicates that there are no propagating modes and only evanescent waves occur, characterized by a pronounced and progressive decay of the energy in this part of the channel due to its lower depth. 
Step up of $10.00 \mathrm{~m}$

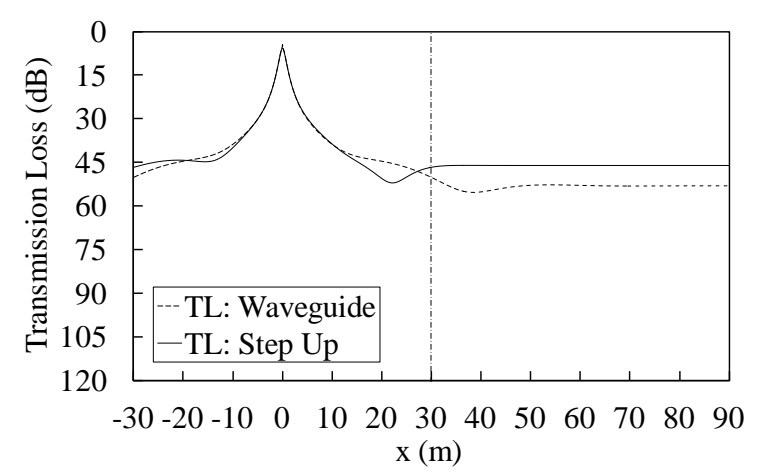

a1)

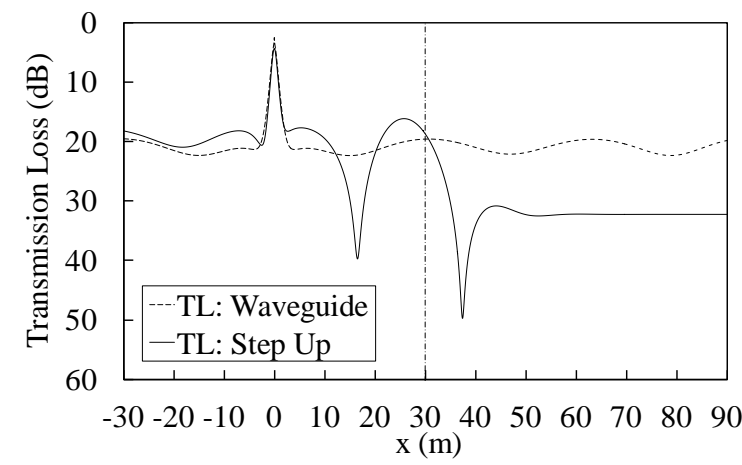

b1)

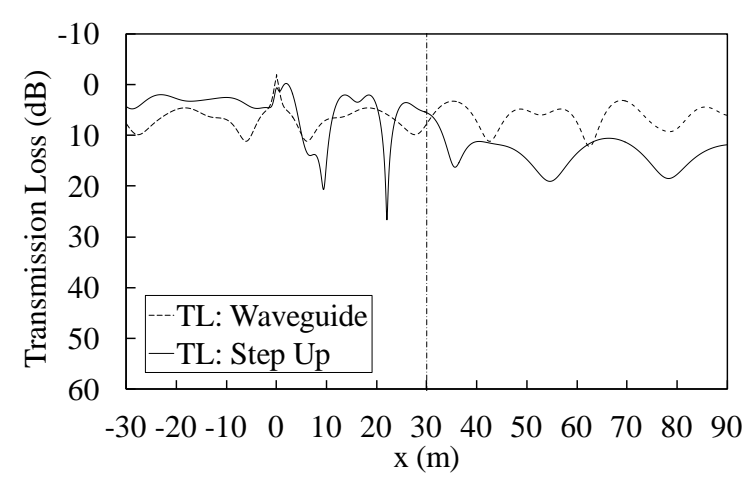

c1)

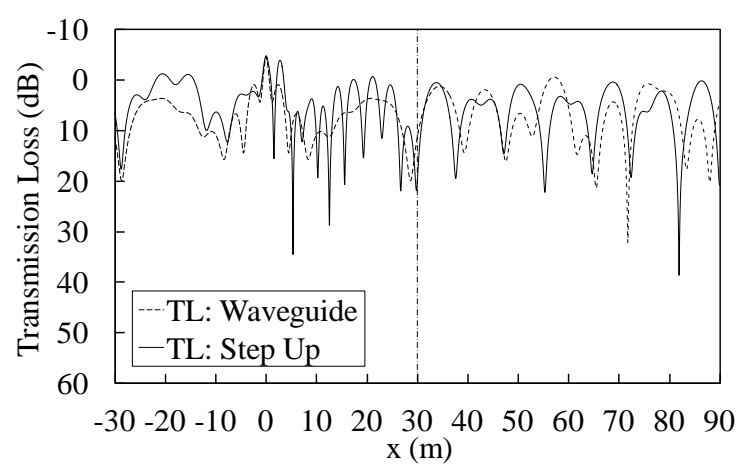

d1)

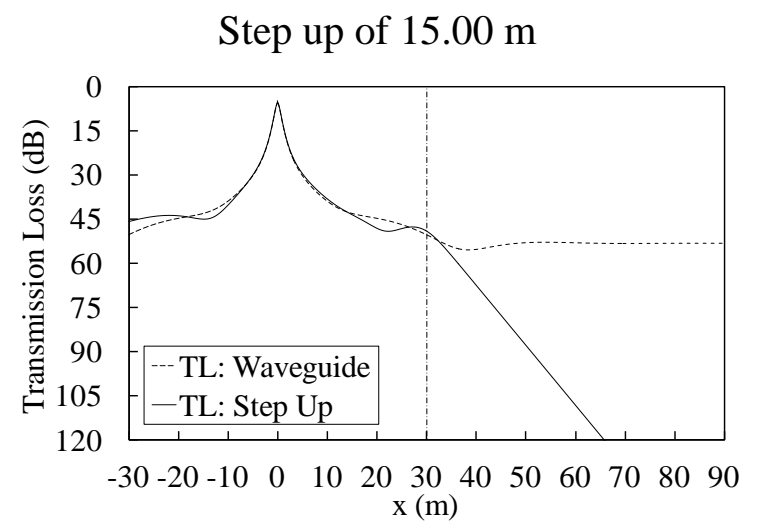

a2)

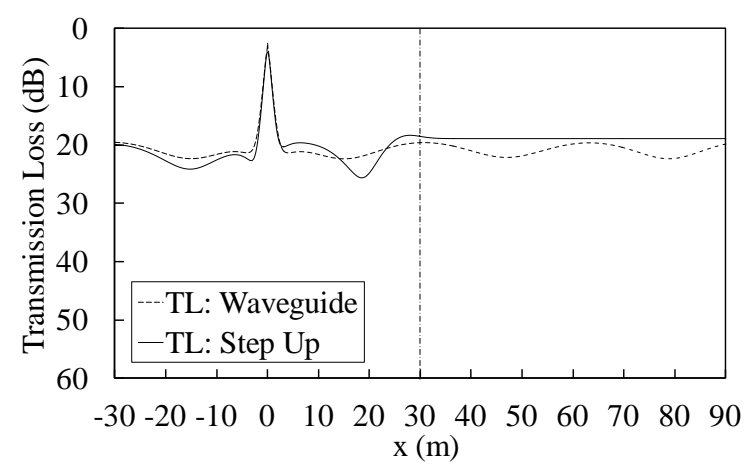

b2)

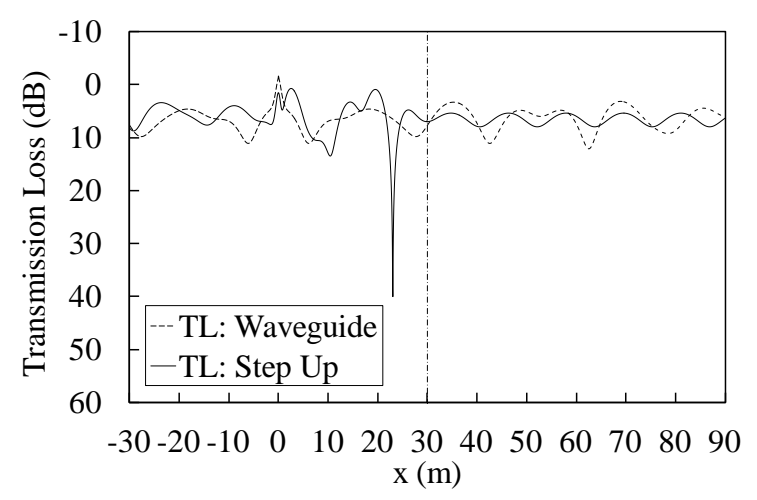

c2)

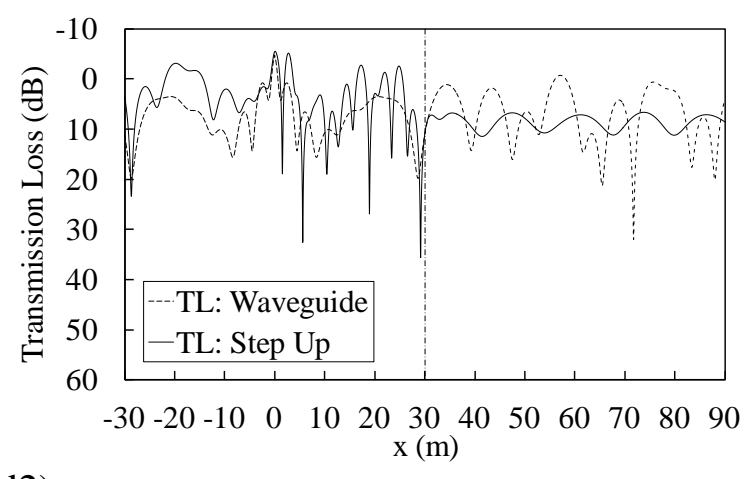

d2)

Figure 15: TL in a waveguide containing a step up with different heights on the bottom of the sea for frequencies of: a) $50 \mathrm{~Hz}$, b) $100 \mathrm{~Hz}$, c) $250 \mathrm{~Hz}$ and d) $500 \mathrm{~Hz}$. 
Time domain responses are computed in shallow water acoustic problems for the identification of important features related to the presence of a step on the seabed, which are not clearly visible in frequency domain analyses. In this work, time domain signals are computed by means of an inverse fast Fourier transform. The pressure field generated by a point source in the spatial-temporal domain is assumed to be defined by a Ricker wavelet. Its Fourier transform is

$U(\omega)=\mathrm{A}\left[2 \sqrt{\pi} t_{0} \mathrm{e}^{-\mathrm{i} \omega t_{s}}\right] \Lambda^{2} \mathrm{e}^{-\Lambda^{2}}$

where $\mathrm{A}$ is the amplitude, $\Lambda=\omega t_{0} / 2 ; t_{s}$ is the time when the maximum occurs, while $\pi t_{0}$ is the characteristic (dominant) period of the wavelet.

The analysis uses complex angular frequencies with $\zeta=0.7 \Delta \omega$ to avoid the aliasing phenomena. In the time domain this shift is later taken into account by applying an exponential window $\mathrm{e}^{\zeta t}$ to the response [20].

A relation of 5 was adopted between the wavelength of the incident waves and the distance between collocation points. The distance between the virtual sources and the collocation points was always 5 times the distance between the collocation points.

The calculations were performed over a frequency range from 2.0 to $1024.0 \mathrm{~Hz}$, assuming a frequency step of $2.0 \mathrm{~Hz}$, which gives a total time of $\mathrm{T}=500 \mathrm{~ms}$. The pressure field was computed over a grid of receivers, equally spaced $\Delta x=0.25 \mathrm{~m}$ and $\Delta y=0.25 \mathrm{~m}$. A sequence of snapshots displaying the pressure field computed within the channel at different time instants is presented to better illustrate the acoustic wave propagation pattern. In all the analyses in the time domain, the responses provided by a flat seabed of constant depth were displayed and used as a reference solution. 
Flat bottom

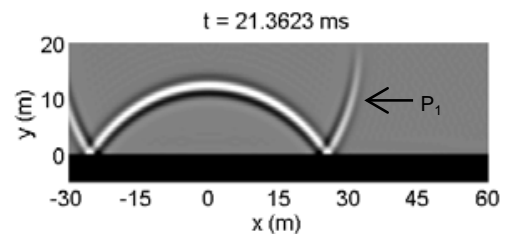

a1)

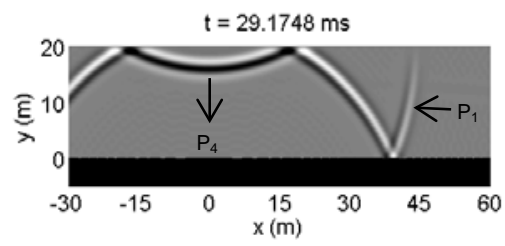

b1)

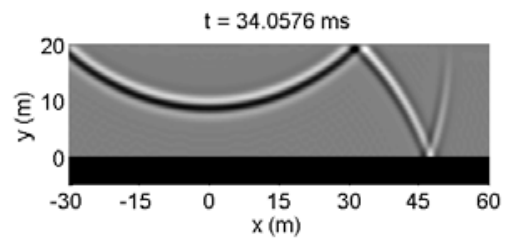

c1)

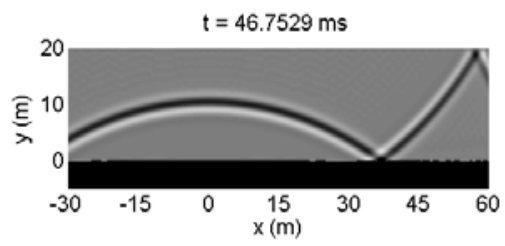

d1)

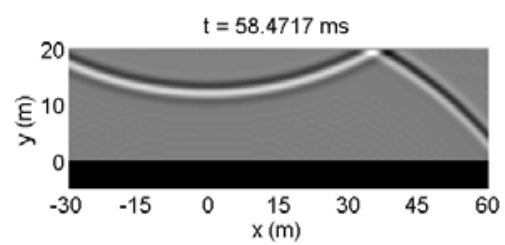

e1)
Bottom with a step of 10.00

$\mathrm{m}$

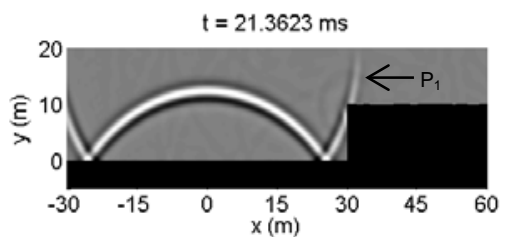

a2)

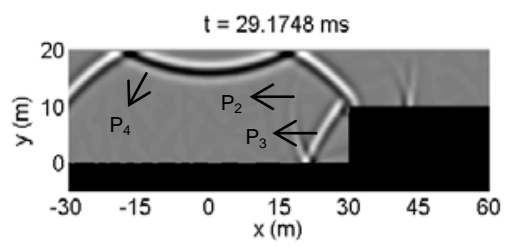

b2)

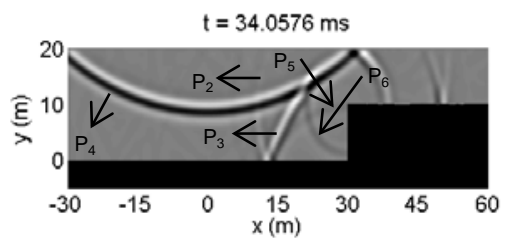

c2)

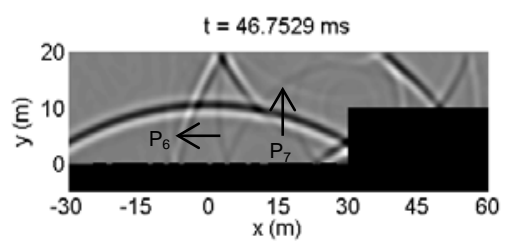

d2)

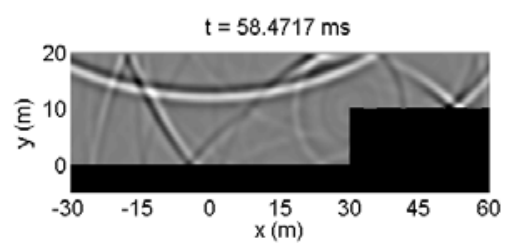

e2)
Bottom with a step of 15.00

$\mathrm{m}$

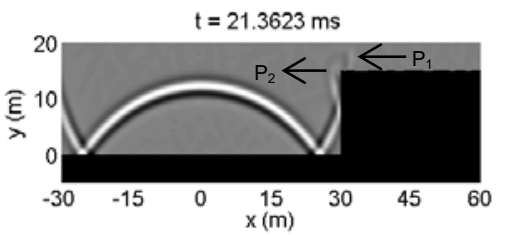

a3)

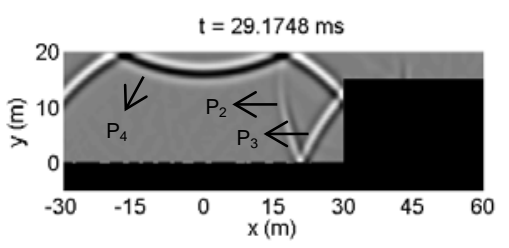

b3)

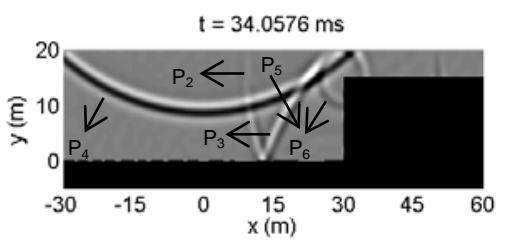

c3)

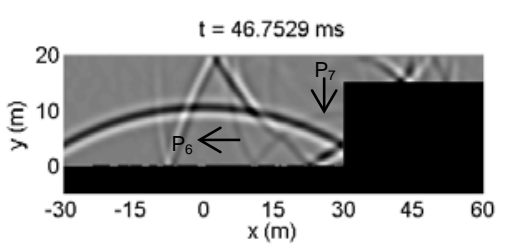

d3)

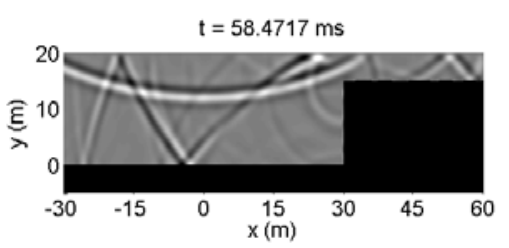

e3)

Figure 16: Time domain responses when a point source excites the channel without and with a step up on the bottom of the sea for different instants: a) $21.3623 \mathrm{~ms}$; b) $29.1748 \mathrm{~ms}$; c) $34.0576 \mathrm{~ms}$; d) $46.7529 \mathrm{~ms}$ and e) $58.4717 \mathrm{~ms}$.

The channel is excited by a point source placed at position $(0.00 \mathrm{~m}, 19.50 \mathrm{~m})$ and the acoustic wave propagation of a Ricker pulse generated by this point source with a characteristic frequency of $400 \mathrm{~Hz}$ is modelled. The first column illustrates pressure distribution snapshots in a waveguide with flat bottom and free surface while the second and third columns depict the plots in the presence of step ups of $10.00 \mathrm{~m}$ and $15.00 \mathrm{~m}$, 
respectively. Figure 16 (a1-a3) displays a snapshot for time instant $t=21.3623 \mathrm{~ms}$, where an incident pulse (P1) can be seen, although it is already combined with a first reflection from the rigid bottom of the sea. In the presence of the step, it is also possible to observe the first reflection from the bottom discontinuity (P2). Later, at time instant $t=29.1748 \mathrm{~ms}$, the second reflection is visible (P3). In addition, at this time, the first reflection generated at the free surface can also be easily identified (P4), with inverted polarity (Figs. 16 (b1-b3)). At time $t=34.0576 \mathrm{~ms}$, a diffracted pulse (P5) with a very low amplitude is generated at the bottom discontinuity (see Figs. 16(c2) and (c3)). In these figures, another diffracted pulse on the top of the step up is also visible (P6). In the plots of Fig. 16(d2) and (d3), at time instant $\mathrm{t}=46.7529 \mathrm{~ms}$, a reflected pulse (P7) in the rigid bottom can also be easily observed. As time passes (Figs. 16(e2) and (e3)), the pulses reflected and diffracted on the surfaces of the channel have generated wave fronts, some propagating towards the higher region of the channel and others propagating towards the lower region of the channel. It is important to note that several wave fronts are generated by reflections on the waveguide, and by reflections and diffractions on the bottom discontinuity between the two regions of the channel, and so, as time elapses, a growing number of reflections and diffractions are registered in the higher region of the channel. This behaviour is more pronounced when a step up of $15.00 \mathrm{~m}$ is considered.

Figure 17 displays the time domain response at receivers placed along a horizontal line at co-ordinates $y=19.00 \mathrm{~m} \quad(x=10.00,20.00,30.00,40.00,50,00 \mathrm{~m})$, to better illustrate the phase inversion each time a pulse hits the free surface. As the distance $x$ increases, the time responses become more complex because of the multiple reflections and diffractions that occur within the channel containing a step up on the bottom.

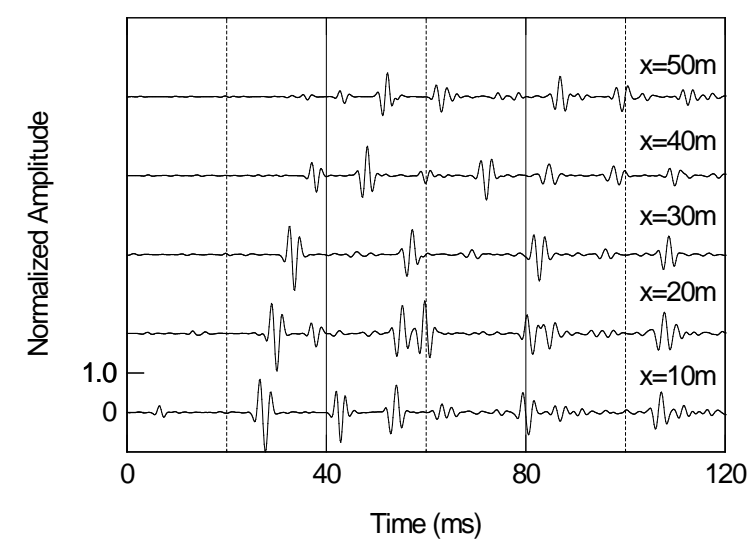

a)

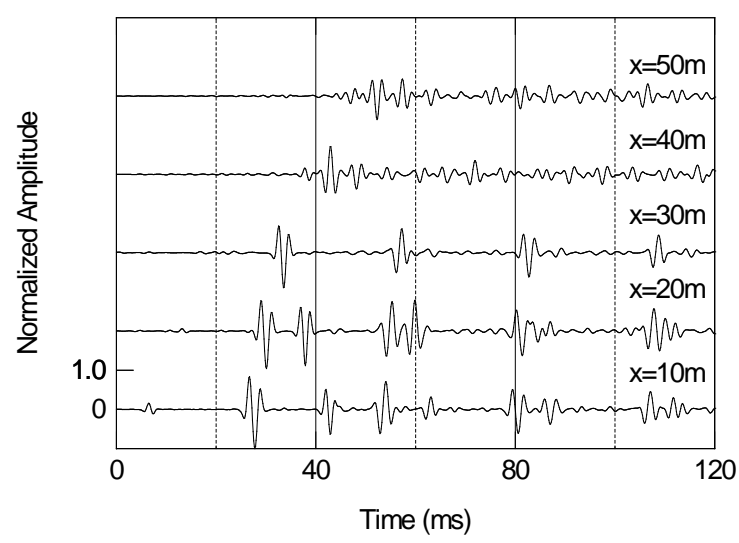

b) 
Figure 17: Time domain response at receivers placed along a horizontal line: a) step of 10.00 $\mathrm{m}$; b) step of $15.00 \mathrm{~m}$.

\section{CONCLUSIONS}

In this paper, the Boundary Element Method and the Method of Fundamental Solutions were used to simulate two-dimensional acoustic wave propagation in a shallow water configuration in the frequency domain, considering a step up on the bottom of the sea. The time domain responses were obtained through an inverse fast Fourier transform of numerical results computed in the frequency domain. Appropriate Green's functions were employed, limiting the required discretization and reducing the computational cost of the proposed models. These Green's functions were obtained either by classic eigenfunction expansion or by Ewald's method. An excellent agreement was obtained between the three proposed models and all three approaches were found to be numerically efficient. In addition, the Green's function's convergence problems that occur when using the boundary integral equation formulation together with the eigenfunction expansion were overcome. The numerical examples demonstrated the use of the proposed models in the analysis of wave propagation in shallow water. In addition, the presented cases of a waveguide containing a step up with different heights, allowed identifying relevant differences in the sound propagation patterns in the underwater acoustic problem in the frequency and the time domain.

\section{Appendix A. Ewald's representation}

The underlying idea of Ewald's representation is to split the integral from 0 to $\infty$ into two parts [12]:

$$
\begin{aligned}
& G_{\mathrm{E} 1}(\boldsymbol{\xi}, \mathbf{x})=\frac{2}{H} \int_{0}^{b^{2}} \frac{1}{\sqrt{4 \pi t}} \sum_{m=1}^{\infty} e^{\lambda_{m} t-\left(\left(x-x_{\xi}\right)^{2} / 4 t\right)} \sin \left[k_{y m}\left(Y_{\mathrm{F}}-y_{\xi}\right)\right] \sin \left[k_{y m}\left(Y_{\mathrm{F}}-y\right)\right] d t, \\
& G_{\mathrm{E} 2}(\boldsymbol{\xi}, \mathbf{x})=\frac{2}{H} \sum_{m=1}^{\infty} \sin \left[k_{y m}\left(Y_{\mathrm{F}}-y_{\xi}\right)\right] \sin \left[k_{y m}\left(Y_{\mathrm{F}}-y\right)\right] \int_{b^{2}}^{\infty} \frac{e^{\lambda_{m} t-\left(\left(x-x_{\xi}\right)^{2} / 4 t\right)}}{\sqrt{4 \pi t}} d t,
\end{aligned}
$$

where the parameter $b$ which divides these integrals is chosen appropriately, taking into account the position of the source and field points [9]. 
In Ewald's approach, the integrals in Eqs. (A.1) and (A.2) are manipulated in order to obtain a Green's function involving rapidly decaying series of special functions. The final expression of the Ewald's representation is:

$$
\begin{aligned}
G_{\mathrm{E}}(\boldsymbol{\xi}, \mathbf{x})= & \frac{1}{4 \pi} \sum_{n=0}^{\infty} C_{n}(\boldsymbol{\xi}, \mathbf{x})+\sum_{m=1}^{\infty} \frac{\cos (\pi m)}{4 \pi} \sum_{n=0}^{\infty} C_{m n}(\boldsymbol{\xi}, \mathbf{x})+ \\
& +\sum_{m=1}^{\infty} \frac{\sin \left[k_{y m}\left(Y_{\mathrm{F}}-y_{\xi}\right)\right] \sin \left[k_{y m}\left(Y_{\mathrm{F}}-y\right)\right]}{2 H \sqrt{-\lambda_{m}}} \times, \\
& \times\left\{\begin{array}{l}
\left.e^{\left(x-x_{\xi}\right) \sqrt{-\lambda_{m}}} \operatorname{erfc}\left[b \sqrt{-\lambda_{m}}+\frac{\left(x-x_{\xi}\right)}{2 b}\right]+\right] \\
+e^{-\left(x-x_{\xi}\right) \sqrt{-\lambda_{m}}} \operatorname{erfc}\left[b \sqrt{-\lambda_{m}}-\frac{\left(x-x_{\xi}\right)}{2 b}\right]
\end{array}\right\}
\end{aligned}
$$

where $\operatorname{erfc}(z)$ is the complementary error function [21] and the coefficients $C_{n}(\boldsymbol{\xi}, \mathbf{x})$ and $C_{m n}(\boldsymbol{\xi}, \mathbf{x})$ are defined as:

$$
\begin{aligned}
& C_{n}(\boldsymbol{\xi}, \mathbf{x})=\frac{k^{2 n} b^{2 n}}{n !}\left[E_{n+1}\left(\frac{a_{1}}{b^{2}}\right)-E_{n+1}\left(\frac{a_{2}}{b^{2}}\right)\right], \\
& C_{m n}(\boldsymbol{\xi}, \mathbf{x})=\frac{k^{2 n} b^{2 n}}{n !}\left[\sum_{j=1}^{4}(-1)^{j} E_{n+1}\left(\frac{a_{j m}}{b^{2}}\right)\right],
\end{aligned}
$$

with $E_{n+1}$ the exponential integral function [21] and the coefficients $a_{1}, a_{2}$ and $a_{j m}$ defined as:

$$
\begin{aligned}
& a_{1}=\frac{\left(x-x_{\xi}\right)^{2}+\left(y_{\xi}+y\right)^{2}}{4}, \\
& a_{2}=\frac{\left(x-x_{\xi}\right)^{2}+\left(-Y_{\mathrm{F}}+y_{\xi}+y\right)^{2}}{4}, \\
& a_{1 m}=\frac{\left(x-x_{\xi}\right)^{2}+\left(2 H m-2 Y_{\mathrm{F}}+y_{\xi}+y\right)^{2}}{4}, \\
& a_{2 m}=\frac{\left(x-x_{\xi}\right)^{2}+\left(2 H m+y_{\xi}-y\right)^{2}}{4}, \\
& a_{3 m}=\frac{\left(x-x_{\xi}\right)^{2}+\left(-2 H m-2 Y_{\mathrm{F}}+y_{\xi}+y\right)^{2}}{4} \text { and }
\end{aligned}
$$


$a_{4 m}=\frac{\left(x-x_{\xi}\right)^{2}+\left(2 H m-y_{\xi}+y\right)^{2}}{4}$.

An appropriate function to define the parameter $b$ was selected based on the following considerations: the parameter $b$ should vary between 0 and $1(0<b<1)$ to accelerate the decay of the term containing $b^{2 n}$, and it should also have a very small value near the singular points. The first singular point is located where the source and field points coincide, i.e. when $r \rightarrow 0$ while the second type of singularity appears when the special function $E_{n+1}\left(a_{2} / b^{2}\right)$ in Eq. (A.4) goes to infinity as the parameter $a_{2}$ in expression (A.5) goes to zero. These singular points are shown in Figure A.1.

For the variation of the field point $\mathbf{x}$, with co-ordinates $(x, y)$, in a vertical line containing the fixed source point $\xi$, with co-ordinates $\left(x_{\xi}, y_{\xi}\right), b(\beta)$ can be defined in the following form:

$b(\beta)=\frac{\alpha}{2}[\cos (\beta \pi)+1]+\gamma$,

where $\alpha$ and $\gamma$ are constants and $\beta$ is a value that depends on the depth $y$ of the waveguide, as illustrated in Fig. A.1. Considering the general case (two singular points), $\beta$ can be defined as [9]:

$\beta= \begin{cases}1+\frac{r}{\beta_{0}} & \text { for } y_{\xi}-\beta_{0} \leq y \leq y_{\xi}+\beta_{0} \\ 1+\frac{a_{2}}{\beta_{0}} & \text { for } Y_{\mathrm{F}}-y_{\xi}-2 \sqrt{\beta_{0}} \leq y \leq Y_{\mathrm{F}}-y_{\xi}+2 \sqrt{\beta_{0}} \\ 0 & \text { for } y \text { outside the above intervals }\end{cases}$

with $\beta_{0}=H / \theta$ being $\theta$ a constant and $r=\left|y-y_{\xi}\right|$. The constants $\alpha, \gamma$ and $\theta$ are assigned empirical values. 


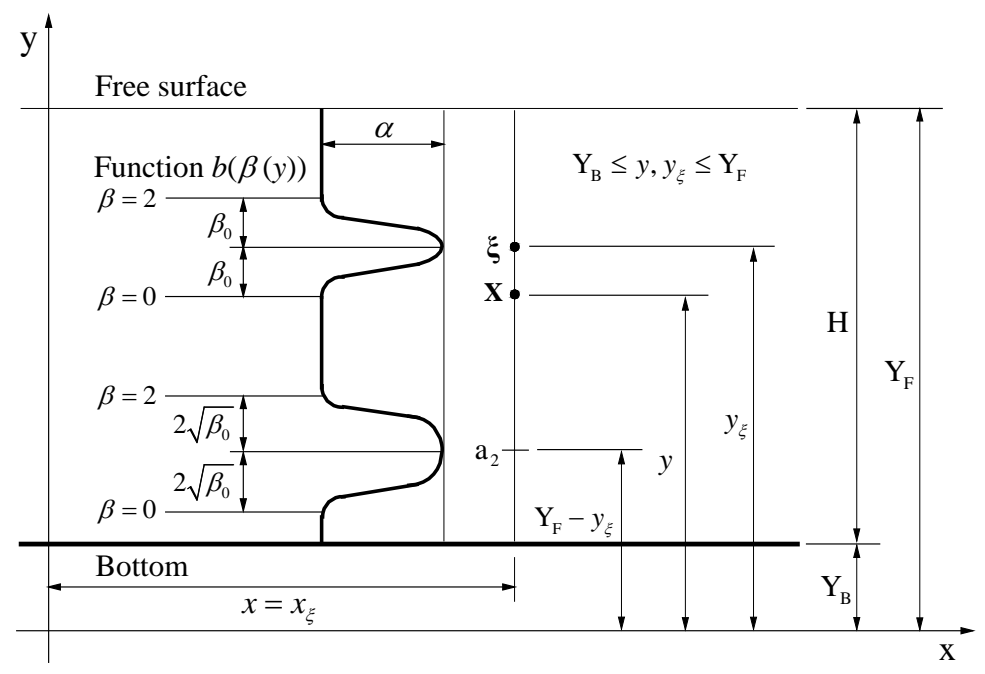

Figure A.1: Function used as parameter $\beta$, considering source and field points located on the same vertical alignment.

\section{ACKNOWLEDGMENT}

The authors would to like thank CNPq (Conselho Nacional de Desenvolvimento Científico e Tecnológico) and FAPERJ (Fundação de Amparo à Pesquisa do Estado do Rio de Janeiro) for providing financial support to this research.

\section{REFERENCES}

[1] Jensen, F. B., Kuperman, W. A., Porter, M. B., Schmidt, H., Computational Ocean Acoustic, AIP Press, Springer, New York, 2000.

[2] Westwood, E. K., Acoustic Propagation Modeling in Shallow Water using Ray Theory, Applied Research Laboratories, The University of Texas at Austin, ARL-TR-89-6, 1989.

[3] Williams, A. O., Normal-mode Methods in Propagation of Underwater Sound, in Underwater Acoustics, ed. R. W. B. Stephens, Wiley-Interscience, New York, pp. 2356, 1970.

[4] Tappert, F. D., The parabolic approximation method, in Wave Propagation and Underwater Acoustics, eds. J. B. Keller and J. S. Papadakis, Springer-Verlag, Berlin, pp. 224-287, 1977. 
[5] Brebbia, C. A., Telles, J.C.F. and Wrobel, L.C., Boundary Element Techniques. Springer-Verlag, Berlin, 1984.

[6] Wu, T. W., On Computational Aspects of the Boundary Element Method for Acoustic Radiation and Scattering in a Perfect Waveguide. J. Acoust. Soc. Am., vol. 96, pp. 37333743, 1994.

[7] Santiago, J. A. F., Wrobel, C., A Boundary Element Model for Underwater Acoustics in Shallow Waters. Comput. Model. Eng. Sci., vol. 1, pp. 73-80, 2000.

[8] Santiago, J. A. F., Wrobel, L. C., Modified Green's Functions for Shallow Water Acoustic Wave Propagation. Engineering Analysis with Boundary Elements, vol. 28, pp. 1375-1385, 2004.

[9] Santiago, J. A. F., Wrobel, L. C., An Efficient Green's Function for Acoustic Waveguide Problems. Communications in Numerical Methods in Engineering. vol. 23, pp. 703-719, 2006.

[10] Linton, C. M., The Green's Function for the Two-dimensional Helmholtz Equation in Periodic Domains. J. Eng. Math., vol. 33, pp. 377-402, 1998.

[11] Linton, C. M. Rapidly Convergent Representations for Green’s Functions for Laplace's Equation. Proc. R. Soc. A., vol. 455, pp. 1967-1997, 1999.

[12] Papanicolaou, V., Ewald's Method Revisited: Rapidly Convergent Series Representation of Certain Green's Functions. J. Comput. Anal. Appl., vol. 1, pp. 105114, 1999.

[13] Venakides, S., Haider, M. A., Papanicolaou, V., Boundary Integral Calculations of Two-dimensional Electromagnetic Scattering by Photonic Crystal Fabry-Perot Structures. SIAM J. Appl. Math., vol. 60, pp. 1696-1706, 2000.

[14] Karageorghis, A. A Practical Algorithm for Determining the Optimal Pseudo-Boundary in the Method of Fundamental Solutions. Advances in Applied Mathematics and Mechanics, vol. 1, n. 4, pp. 510-528, 2009. 
[15] Costa, E. G. A., Godinho, L., Santiago, J. A. F., Pereira, A., Dors, C., Efficient Numerical Models for the Prediction of Acoustic Wave Propagation in the Vicinity of a Wedge Coastal Region. Engineering Analysis with Boundary Elements, vol. 35, pp. 855-867, 2011.

[16] Costa, E. G. A. Godinho, L., Pereira, A., Santiago. J. A. F., Prediction of Acoustic Wave Propagation in a Shallow Water Configuration using the Method of Fundamental Solutions. Journal of Computational Acoustics, vol. 20, n. 4, 1250013 (34 pages), 2012.

[17] Pederson, T. K., Modelling Shallow Water Acoustic Wave Propagation. M.Sc. Thesis. University of Rhode Island, USA, 1996.

[18] Tadeu, A., Santos, P., Kausel, E., Closed-from Integration of Singular Terms for Constant, Linear, Quadratic Boundary Elements - Part 1: SH Wave Propagation, Engineering Analysis with Boundary Elements, n. 8, vol. 23, pp. 671-681, 1999.

[19] Tadeu, A., Santos, P., Kausel, E., Closed-from Integration of Singular Terms for Constant, Linear, Quadratic Boundary Elements - Part II: SV-P Wave Propagation, Engineering Analysis with Boundary Elements, n. 9, vol. 23, pp. 757-768, 1999.

[20] Kausel, E., Roesset J. M., Frequency Domain Analysis of Undamped Systems. Journal of Engineering Mechanics ASCE, n. 118, pp. 721-734, 1992.

[21] Abramowitz, M., Stegun, I. A., Handbook of Mathematical Functions with Formulas, Graphs and Mathematical Tables. New York, Dover Publications, 1965. 